\title{
XIV.
}

\section{Ueber einen Fall von Worttanbheit und das Lichtheim'sche Krankheitsbild der subcorticalen sensorischen Aphasie.}

\author{
Von \\ Dr. Franz Ziehl \\ in Lübeck. \\ (Mit 3 Abbildungen.)
}

„Bei der Aphasie wird das Experiment nicht vom Experimentator, sondern von der Natur angestellt, so dass die Beobachtung der ausschlaggebenden Versuche nicht in das Belieben des Untersuchenden gestellt, sondern von einem glücklichen Zufall abhängig ist. Deshalb kann der Ausbau des Gebäudes nur langsam fortschreiten, er kann nicht von einem Einzelnen geleistet werden, sondern muss aus der gemeinsamen Arbeit Vieler hervorgehen." Diese Bemerkungen, die einem ausgezeichneten, sehr bekannt gewordenen Aufsatz Lich theim's 1) uiber Aphasie entnommen sind, legen es Jedem nahe, der einen Fall von Aphasie zu sehen Gelegenheit hat, denselben nicht nur möglichst genau zu beobachten, sondern ihn dann auch durch Veröffentlichung der Wissenschaft zu erhalten, wenn der Fall klinisch einfach und uncomplicirt ist und in seinem Symptomencomplex etwas zeigt, was bisher nicht oder nur selten beschrieben worden ist.

Ein solcher Fall scheint mir der unten geschilderte zu sein. Er ist uncomplicirt; denn die Worttaubheit trat bei völliger geistiger Gesundheit plötzlich auf, und das klinische Bild blieb sich vom Anfang bis zu dem aus anderer Ursache erfolgten Tode völlig gleich. Der Kranke gehörte den besseren Ständen an, was die Beobachtung in solchen Fällen, wo es auf die Intelligenz ankommt, wesentlich erleichtert. Die Punkte, die dabei besonders zu beriicksichtigen sind, waren mir ausserdem geläufig, da ich seit einer Reihe von Jahren, angeregt durch das Werk Preyer's über die Entwicklung der Seele des Kindes, an meinen eigenen Kindern und an mir selbst allerlei

1) Ueber Aphasie. Deutsches Archiv f. klin. Medicin. Bd. XXXVI, S. 204. 
Sprachstudien angestellt hatte, auf die ich bei passender Gelegenheit vielleicht einmal eingehen werde, da sie mir einige interessante Streiflichter auf die Natur der Sprachstörungen zu werfen scheinen. Demnach dürfte in dern nachstehenden Falle nichts Wesentliches ubersehen worden sein. Ich gebe zunächst die Krankengeschichte und zwar möglichst ausführlich; denn wie sich später zeigen wird, können solche Beobachtungen gar nicht genau genug mitgetheilt werden, da immer wieder neue theoretische Fragen auftauchen, deren Lösung oft unmöglich ist, weil man in früheren Fällen keine entsprechenden Angaben findet. Ich hoffe somit, wenn auch die ansehliessende theoretische Analyse des Falles bei manchen auf Widerspruch stossen dürfte, dennoch auch diesen wenigstens einen willkommenen casuistischen Beitrag zur Lehre von der Aphasie gegeben zu haben; denn es scheint mir trotz der grossen Casuistik an gründlich beobachteten, möglichst klaren und einfachen Fällen immer noch Mangel zu herrschen.

Wer iibrigens die zugehörige Literatur der letzten Jahre genauer verfolgt hat, dem wird es nicht entgangen sein, dass in dieser Frage bei uns in Deutschland der bisherige etwas exclusive Standpunkt von der Annahme bestimmter, stets vorhandener anatomischer Sprachcentren verschiedener Function, die stets durch bestimmte Leitungsbahnen bei allen Menschen in gleicher Weise verbunden sind, einer mehr physiologisch-psychologischen Auffassung wird weichen müssen, die zugleich individuellen Verschiedenheiten mehr Rechnung trägt.

\section{Eigener Fall und dessen Analyse.}

Patient, ein 75 jahriger noeh sehr rïstiger Kaufmann, seit Jahren an Nierengries leidend, im Uebrigen aber gesund, sitzt am 24. December 1891 Nachmittags gemuithlich plaudernd beim Kaffee, als seine Tochter bemerkt, dass ihr Vater die Cigarre verkehrt in den Mnd nimmt. Sie macht ihn darauf aufmerksam, er antwortet aber nicht. Als sie ihn nun anstösst, nimmt er zwar die Cigarre sofort aus den Mund, eine mündliche Verständigung aber ist unmöglich, obschon der Kranke selbst vernünftig spricht. Der sofort herbeigerufene, ganz nahebei wohnende Hausarzt stellt fest, dass Patient das Vermögen, Worte zu verstehen, gänzlich verloren hat, dagegen Geräusche u. s. w. vollkommen deutlich hört. Patient spricht verständlich, auch ganz logisch, verwechselt aber Buchstaben und Silben beim Sprechen und verstiummelt einzelne Worte. Lesen kann er mit Verständiss; denn die ganze Unterhaltung muss schriftlich geschehen. Irgend eine Lähmung war nicht vorhanden.

Am 6. Jantar 1892 sah ich den Kranken, an dessen Zustand sich nichts geändert hatte. Er ist gerade eifrig mit Zeitungslesen beschäftigt. Das Sensorium vollkommen frei. Das Gedächtniss ganz intact; denn er weiss nach Angabe seines Sohnes tiber alle Einzelheiten seines Geschäfts 
Bescheid, kennt die Tage, an denen Wechsel fällig sind, die betreffenden Summen a. s. w.; die Intelligenz ganz erhalten.

Sensibilität, Motilität, Reflexe durchaus normal, keine Hemiopie. Die Hörprüfungen ergeben, vom Sprachverständniss abgesehen, durchaus gute Hörschärfe, W e ber'scher und Rinne'scher Versuch wie beim Gesunden. Eine ausklingende Stimmgabel wird sowohl bei Laft- als bei Knochenleitung fast so lange gehört wie von mir selbst, die geringe Differenz ist wohl dem Alter des Patienten zazuschreiben. Tiefe und hohe Töne hört er gleich gut, keine ausfallenden Töne. Im rechten Trommelfell einige kleine Verkalkungen, sonst ergiebt die Ohrenspiegeluntersuchung nichts Abnormes.

Der nachfolgende Befund über die Sprachverhältnisse ist das Ergebniss zahlreicher, oft wiederholter Untersuchungen von stets gleichem Resultat. Irgend eine Veränderung des Zustandes vom Beginn der Erkrankung bis zu dem am 23. Juni 1892 erfolgten Tod ist nicht aufgetreten. Der Tod trat infolge von Harnverhaltung unter urämischen Erscheinungen nach kurzer Krankheit ein. Die Section wurde leider nicht gestattet.

Verständniss für die Sprache und für Musik: er versteht keine an ihn gerichtete Frage. Er hört zwar, dass gesprochen wird, kann aber den Sinn der Worte nicht auffassen. Scheinbar versteht er mitunter ein Wort. Die genaue Beobachtung zeigt aber, dass dies nur durch Ablesen von den Lippen des Sprechenden geschieht. Er versteht auch nichts, wenn man ihm möglichst deutlich und mit lauter Stimme Worte direct in das Ohr, sei es das rechte, sei es das linke, hineinspricht. Stets ist seine Antwort, ,ich höre wohl, ich höre alles, aber es ist Wirrwarr", ,alles geht mir durcheinander", „es ist ein wirres Gewoge in meinem Ohr" u. dergl. Bemerkenswerth ist, dass er überhaupt meist gar nicht Acht giebt, wenn gesprochen wird; er giebt sich auch gar nicht einmal die Mühe zuzuhöıen. Es kommt daher auch gar nicht vor, dass er, wie z. B. Schwerhörige oder andere sensorisch Aphasische Worte missversteht, er antwortet uiberhaupt niemals auf etwas. Er achtet auf das Sprechen nur dann, wenn ihm schriftlich mitgetheilt wird, er möge aufpassen, man wolle mit ihm sprechen, um sein Gehör zu prïfen. Jeder würde ihn daher wohl für taub halten, wenn nicht seine gegentheiligen Versicherungen und die weitere Prüfung ergäbe, dass er ebenso gut hört, wie ein gesunder Mensch. Er hört alle Geräusche, z. B. das Uhrticken, das Klingeln einer Glocke, das Bellen seines Hundes, Händeklatschen, das Oeffinen und Schliessen der 'Thür u. s. w. Das Gehörte bezieht er auch ganz richtig auf seine Ursache, z. B. erkennt er das Klingeln der Hausglocke und unterscheidet es von dem Klingeln anderer Glocken. Er erkennt sogar schon von Weitem das Kommen seines Arztes an dem Rollen des Wagens auf der Strasse, indem er dies genau von dem Rollen anderer Wagen unterscheidet, ,ich höre Alles ganz fein, aber mit der Sprache ist es eine merkwïrdige Geschichte". An der specifischen Klangfarbo ihrer Sprache kann er daher aich im Nebenzimmer sprechende, ihm bekannte Personen erkennen. - Töne hört er ebenso gut wie Geräusche, er unterscheidet sie richtig nach ihrer Höhe und Tiefe, erkennt jedes Instrument an seiner Klangfarbe, indem er z. B. Töne eines Claviers von denen einer Stimmgabel, einer Drehorgel u. s. w. unterscheidet. 
Ohne sein Wissen im Nebenzimmer ertönendes Clavierspiel hört er sofort ,da wird ja Clavier gespielt, das höre ich gar zu gern; aber dies Stüek ist mir zu sehwer, das versteh ich nicht, meine Tochter soll aus der ,Martha' spielen". Er erkennt jedoch auch hiervon nichts, nicht einmal das bekannte "letzte Rose“, sondern meint „nein, es läuft Alles durcheinander, das Verständniss fehlt mir, die Musik ist auch verwirrt, die Töne kommen mir alle durcheinander". Auch die einfachsten, ihm ganz geläufigen Melodien, z. B. „o Tannenbaum" u. dergl. erkennt er nicht, auch dann nicht, wenn nur die Melodie allein ohne Begleitung auf dem Clavier gespielt wird, „es ist mir ganz unmöglich, das zu verstehen". -

Fir hört und erkennt einzelne ihm vorgesprochene Buchstaben, z. B. die Vocale und Doppelvocale sämmtlich, die Consonanten, wenn man sie deutlich spricht; denn schriftlich aufgefordert, sie nachzusprechen oder aufzuschreiben, thut er es. Das Verständniss ist aber nur dann vorhanden, wenn man die Consonanten stumm als Geränsche spricht, nicht wenn man sie mit ihrem Namen nennt; also z. B. $r$ als „rrr", nicht als ,er"; s als "sss", nicht als „es". Spricht man erst „r"r, dann gedehntes „o", so wird beides allein richtig erkannt und nachgemacht, aber beides zusammen „,roh" wird nicht erkannt, man mag den Uebergang vom $r$ zum o noch so langsam und deutlich machen. Es kann auch nicht nachgesprochen werden ,ich bin dazu nicht im Stande, ich höre es, aber nachmachen kann ich es nicht. Soll es ,Fenster heissen? Nein, das ist's nicht, das ist mir doch anders im Ohr, (nach einigem weiteren Besinnen) ich weiss wirklich nicht, was Sie gemeint haben". Genau so geht es mit a und 1 und verbunden als "Aal" $\mathrm{u}$. dgl. Er erkennt demnach die Vocale an ihrer Höhe und Klangfarbe wohl wie er andere Töne erkennt, die Consonanten als Geräusche wie er andere Geräusche hört. $\mathrm{Zu}$ Wörtern zusammengefügte Buchstaben aber sind seinem Verständniss verschlossen, da das zugehörige Wortklangbild bei ihm nicht entsteht.

Sprache: Patient spricht viel und gern. Er spricht in fliessender Rede durchaus verständig und vernïnftig, verwechselt jedoch mitunter Buchstaben und Silben, auch ähnlich klingende Wörtcr, zuweilen, aber am seltensten, werden auch ganz anders klingende Wörter statt der gewollten gebraucht. Doch ist die Paraphasie nur von mässigem Grade, man versteht immer sofort, was gemeint ist, z. B. „Spiel mal aus der ,Zauberflaute' oder aus ,Don Wan', weich' mir die Hand mein Leben." - „Ich habe heute etwas ,Kopfschwarz', meine ,Vernachten' (Verwandten) waren zu lange hier, da habe ich meine ,Stumme ${ }^{6}$ zu sehr angestrengt und $\mathrm{zu}$,stiel" gesprochen." - "Ich muss leute wegen meiner Niere wieder 12 ,Falschen" Wildunger haben." — "Der Bleistift ,schraubt" wohl schlecht." - Die Paraphasie ist sichtlich von der ganzen Stimmung des Patienten abhängig, da sie an Tagen, wo er körperlich nicht ganz wohl ist (z. B. gelegentlich bei einem Schnupfen), mehr hervortritt, als an anderen: - Auf Verlangen pfeift er eine ihm bekannte Melodie richtig vor.

Nachsprechen: Wie oben bereits bemerkt, kann er auch die einfachsten Wörter nicht nachsprechen, wohl macht er leicht nachzuahmende Geräusche nach, z. B. Brummen, die Buchstabengeräusche, Töne (auch richtig nach ihrer Höhe); ferner Onomatopoëtika, z. B. „wau wau“. 
Lachend sagt er bei dem Versuch: „Sie bellen ja grade wie mein Hund“. Andere derartige Wörter aber versteht er nicht, z. B. „miau", und kann sie nicht nachmachen, vermuthlich weil sie ihm nicht so geläufig sind wie gerade das Bellen seines eigenen Hundes.

Schriftverständniss: Sowohl Geschriebenes als Gedrucktes versteht er richtig, wie schon daraus hervorgeht, dass die ganze Verständigung mit ihm schriftlich geschehen muss.

Schrift: Die Fähigkeit zu schreiben ist erhalten, die Schrift ist sogar richtiger als die Sprache, da weniger Fehler (Verwechslungen von Silben und Buchstaben) vorkommen als beim Sprechen. Hierauf machten die Verwandten des Patienten mich zuerst aufmerksam, die darüber sehr erstaunt waren, und ich habe mich davon an von ihm herriihrenden Briefen - besonders solche aus der späteren Zeit - wiederholt auf das Bestimmteste iberzeugt, z. B. fanden sich in einem längeren Schreiben nur folgende drei Fehler Cantrotiania (Christiania), Leist (Zeit) und Conto aufstreiben (offenbar aus aufstellen und aufschreiben zusammengeflossen). Die Handschrift ist wie früher.

Dictatschreiben: Ist natürlich unmöglich, da das Sprachverständniss fehlt. ')

Lautlesen: Besser als die freie Rede, da die Paraphasie auffallend geringer ist, oft fehlt sie ganz. Er liest jedoch auffallend langsam und bedächtig. Bei freier Rede folgen die Wörter viel schneller aufeinander.

Bezeichnen vorgezeigter Gegenstände: Geschieht stets ohne Bedenken richtig, die Wörter fehlen nicht, nur sind mitunter Silben oder Buchstaben darin verkehrt wie beim freien Sprechen.

Verlauf: Der Befund war, wie schon oben bemerkt, von Anfang bis zum Tode der gleiche. Keine weiteren Gehirnsymptome traten hinzu. Besonders zeigten sich auch keine secundären Störungen des Gehirns, wie z. B. Zeichen von Gehirnatrophie, wie sie so häufig zu Gehirnläsionen hinzutreten. Nichts trat ein, was auf allgemeine intellectuelle Schwäche oder Defecte in der Intelligenz, Abnalıme des Gedächtnisses u. s. w. hätte bezogen werden müssen.

Eine kurze Zusammenfassung ergiebt Folgendes: Bei einem 75 jährigen Manne entsteht plötzlich und ohne apoplektischen Insult, nur mit momentaner leichter Verwirrung (nimmt die Cigarre verkehrt in den Mund), ein gänzlicher Verlust des Verständnisses für Sprache und Musik bei völligem Erhaltenbleiben des Gehörvermögens für Geräusche und Töne, die freie Rede zeigt paraphasische Störungen, ebensolche, aber in viel geringerem Grade, weist lantes Lesen und die Schrift auf. Dieses Bild bleibt durch 6 Monate bis zum Tode völlig unverändert. Irgend welche Intelligenz- oder Gedächtnissstörungen sind nicht vorhanden.

Es dürfte nicht schwer sein, auch ohne Sectionsbefund eine voll-

1) Ma lachowski (Sammlung klin. Vorträge von Volkmann, S. 2959) giebt sonderbarer Weise an, dass das Dictatschreiben bei der subcorticalen Aphasie erhalten sei.

Deutsche Zeitschr. f. Nervenheilkunde. VIII. Bd. 
kommen sichere Diagnose zu stellen. Das Hauptsymptom ist ein reines Ausfallssymptom (Verlust des Sprach- und Musikverständnisses), das Gehörvermögen ist völlig unberührt. Um eine Erkrankung des Ohres kann es sich also nicht handeln, auch weist die Paraphasie direct auf eine Gehirnerkrankung hin. Durch sie wird auch die Annahme einer Labyrintherkrankung ausgeschlossen, auch würde man sich schwer eine Vorstellung davon machen können, auf welchem Wege plötzlich und blitzschnell eine solche Erkrankung, die doch doppelseitig sein müsste, hätte zu Stande kommen können. Da Allgemeinsymptome ansser momentaner leichter Verwirrung fehlten, so kann nur eine umschriebene, wenig umfangreiche Läsion vorliegen, die Druck oder Fernwirkungen nicht erzeugt hat. Da das Bild ein ganz stationäres blieb, kann es sich auch nicht urn einen fortschreitenden Process gehandelt haben, sondern nur um ein plötzliches Ereigniss. Zweifellos diurfte ein kleiner Blutungsherd vorgelegen haben, dessen directe oder doch directen so gut wie grleichstehende, weil von unmittelbarer Nachbarschaft herrihrende, indirecte Symptome die Worttaubleit und die Paraphasie sind. Bei der Einfachbeit des ganzen Krankheitsbildes muss man es bedauern, dass die Section nicht gemacht werden konnte, da sie einen interessanten Beitrag zu Localisationsfrage geliefert hätte. Indessen auch ohne eine solche erlaubt der Fall allerlei klinische und psychologische Schlüsse, für deren Sicherheit die fehlende Section völlig gleichgïltig ist. Nach Lichth eim's Darstellung bätte die Läsion im weissen Marklager des linken Schläfenlappens, dort wo beide Acustici in denseiben einstrahlen, sitzen müssen. Dass sie wenigstens links sass, beweist die Paraphasie, die nur durch einen linksseitigen Herd zu erkiăren ist.

Um zu einem befriedigenden Einblick in die Eigenthümlichkeiten des vorliegenden Falles zu kommen, wird man ihn am besten an der Hand eines der vorhandenen Schemata betrachten. Wir wählen dazu das bekannte Lichtheim'sche mit besonderer Berücksichtigung der dazu von Wernicke ${ }^{1}$ ) gemachten Zusätze, da wohl allgemeine Uebereinstimmung darüber herrscht, dass dessen Bilder die einfachsten und klarsten sind. Das Schema anzuwenden, ist in unserem Falle ganz besonders angebracht, da Lichtheim ${ }^{2}$ ) in seinem oben erwähnten Aufsatz das vorliegende Krankheitsbild gleichsam vorausberechnet hatte.

Bekanntlich unterscheidet er ein Klangbildcentrum $A$ als den Sitz der akustischen Erimnerungsbilder für die Worte und ein Be-

1) Fortschritte der Medicin. 1885. S. 824,1886 S. 381 u. 463.

2) 1. c. S. 244 . 
wegungsbildcentrum $M$ als den Sitz der Erinnerungsbilder für die beim Aussprechen der Worte ausgeführten Bewegungscomplexe. $\mathrm{Zu}$ $A$ führt die centripetale Bahn $A a$, die die Gehörseindrücke übermittelt, von $M$ geht die centrifugale Bahn $M m$ aus, die die Sprechbewegungen innervirt. Dem Schema soll als Grundlage ein für die Nachahmungssprache des Kindes angeblich nothwendiger Reflexbogen ${ }^{1)}$ a AMm dienen. Taucht beim Kinde das Verständniss für die Bedeutung der nachgeahmten Worte auf, so muss sich eine Verbindung zwischen $A$ und einem hypothetisehen Centrum für die Bildungsstätte der Begriffe $B$ herstellen. Für die willkürliche Sprache construirt Lichtheim eine centrifugale directe Bahn $B M$, während $\mathrm{Kussmaul}$ dafür bekanntlich nur eine indirecte, also über $A$ führende Bahn $B A M$ zulässt. Ferner nimmt $L i c h$ theim ein Centrum $O$ für die optischen Erinnerungsbilder an die Buchstaben und an die Buchstabencomplexe an. Da Lesen und Schreiben im Uebrigen in engem Anschluss an die Sprache erlernt wird, so sollen zum Theil die gleichen Bahnen wie für die Sprache dazu dienen. Das Verständniss für die Schrift vermittelt die Bahn $O A B$, das laute Lesen erfolgt über $O A M m$. Ein Centrum $E$ innervirt die Schreibebewegungen, durch die Bahn $O E$ ist es

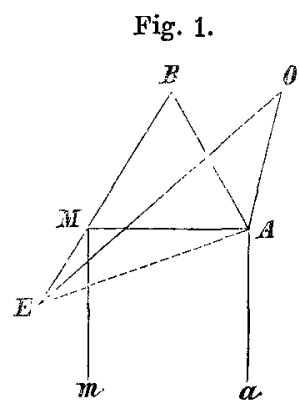

Schema Lichtheim's. mit den optischen Schriftbildern verknüpft; durch deren Vermittlung erfolgt das (automatische) Nachmalen von Buchstaben. Das willkturliche (begriffliche) Schreiben erfolgt über $M$, ob auch zugleich über $A$, ist für Lichtheim zweifelhaft, also entweder über $B M E$ oder über $B M A E$.

Wernicke hat das Lichtheim'sche Schema mit folgenden Abweichungen adoptirt, die besonders aus der Einführung des „Wortbegriffs" sich ergeben. Der Begriff eines Wortes an sich nur als Complex verschiedener Laute aufgefasst, ohne Bezug auf die dem

1) Die Einführung des Wortes Reflex an dieser Stelle und die Auffassung des Vorganges als eines Reflexvorganges halte ich nicht für besonders glücklich. Unter Reflex versteht man sonst etwas vom Willen Unabhängiges, höchstens dadurch Hemmbares. Die ganze Auffassung der Sprachvorgänge beim Kinde wird hierdurch meines Erachtens von vornherein eine falsche, dem wirklichen Vorgang durchaus widersprechende, wenigstens soweit ich das nach Beobachtungen an meinen eigenen Kindern beurtheilen kann. Die Kinder verstehen bereits manche Wörter und sprechen freiwillig allerlei Laute und Silben, während es ihnen ganz unmöglich ist, ihnen nicht geläutige Silben, selbst solche mit denselben Buchstaben, nur in anderer Reihenfolge nachzusprechen. Hierin hat Preyer vollkommen recht. 
Wort zukommende begriffliche oder sinnliche Vorstellung, besteht nämlich nach ihm aus den fest associirten Erinnerungsbildern in $M$ und $A$. Beim Verstehen der Sprache erregt der erste Act den Begriff des Wortes, der zweite den des zugehörigen Gegenstandes. Beim spontanen Sprechen taucht zuerst der Begriff des Gegenstandes, dann der des Wortes auf. Das Klangbild genügt nun, um den Begriff zu erregen; diese Association ist fest und selbständig. Umgekehrt dagegen ist diejenige zwischen Begriff und der betreffenden Wortbewegungsvorstellung weniger fest und nicht ausreichend, das correcte Sprechen zu sichern. Dazu gehört die Innervation des ganzen Wortbegriffs $M+A$. Ist $A$ zerstört oder die Bahn $M A$ ungangbar, dann entsteht nach Wernicke Paraphasie. Er erklärt dies dadurch, dass die ersten Sprachkenntnisse des Kindes im Verstehen der Wörter beständen, also in der Association von Klangbildern mit concreten Begriffen, während die Fertigkeit des Sprechens erst viel später sich

Fig. 2.

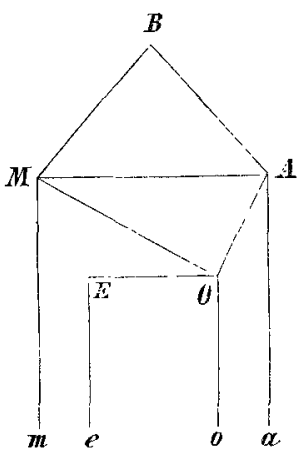

Schema Wernicke's. entwickle. Eine Vorstufe dieser letzteren sei die Fähigkeit, durch Bentitzung der Bahn $M A$ die zugehörigen Sprachklänge nachzusprechen. Aus diesem Grunde stellt er sich die centrifugale Innervation des Wortbegriffs ron Seiten des concreten Begriffs als auf doppeltem Wege erfolgend vor, auf dem einfachen Wege $B M$ und dem complicirteren $B A M$.

Auch nach Lich theim ist für das correcte Sprechen die Mitwirkung der Klangbilder erforderlich. Er lässt aber deren Innervation auf dem Wege $B M A$ erfolgen, und nun soll das Klangbild selbst wieder noch mit dem Begriff in Verbindung gebracht werden auf dem Wege $A B$. Auf dieses Mitinnervirtwerden der Klangbilder beim Sprechen ist er durch Selbstbeobachtung gekommen. Wenn er die zur Aussprache eines Wortes nothwendige Mundstellung herstelle, ohne das Wort auszusprechen, so ertöne bei ihm das betreffende Klangbild aufs deutlichste. Für die fliessende Sprache sei jedoch im Allgemeinen die Innervation der Klangbilder nicht nöthig. Es genlige die Bahn $B M m$. Beim Benennen eines Gegenstandes aber werde das uber $M$ in $A$ erregte Klangbild den Umweg durch $B$ machen, um die wirkliche Innervation der Sprachbewegung herbeizuführen. Das gefundene Klangbild werde somit noch einmal über die Schwelle des Bewusstseins gehoben. Unter Umständen soll sich indessen auch die fliessende Sprache in ähnlicher Weise vollführen. Paraphasie 
entsteht also nach Lichtheim, wenn in dem Kreise $B M A B$ eine Unterbrechung in der Weise statt hat, dass die Sprache dadurch nicht vollkommen gehemmt wird.

Als beweisend fur seine Anschaung führt $\mathrm{Lich}$ theim an, dass Kranke, deren Centrum $M$ zerstört sei, die Wörter innerlich nicht mehr erklingen lassen könnten; denn sie seien nicht im Stande, die Silbenzahl von Wörtern, welche vorgezeigten Objecten entsprächen, anzugeben, folglich geschehe die Innervation der inneren Klangbilder iiber $M$. Seine Deutung nennt Lichtheim selbst eine etwas complicirte. Da er nur Wortklangbilder als innere Worte kennt, da seine Bahnen ferner alle nur in einer Richtung leiten, so ist seine Anschauung in sich vollkommen consequent. Indessen hat sich noch Niemand, soweit ich sehe, für sie erwärmen können; wohl jeder, der sich die Sache überlegt, wird die Empfindung haben, das Schema führt hier zu einer Unmöglichkeit. Der Fehler kann bei der inneren Consequenz desselben dann nur in den Principien liegen, und so ist auch Wernicke hierin anderer Ansicht, indem er meint, die erst spät erworbene Zerlegung des Wortes in Silben könne nur am Wortbegriff geschehen. Dieser aber fehle den erwähnten Patienten Lich t heim's wegen Zerstörung von $M$. Daher sei sein Experiment nicht beweisend. Die Bahn $B A$ müsse doppelseitig auch noch aus dem Grunde leiten, weil in gewissen Fällen zwar nicht spontan gesprochen, wohl aber mit Willen nachgesprochen werden könne und dem Willensimpuls bleibe, um nach $M$ zu gelangen, kein anderer Weg als $B A M$.

Neuere Schriftsteller über Aphasie sind ganz zu der Kuss maulschen Darstellung zurückgekehrt und lassen $M$ nur über $A$ innervirt werden; alsdann entsteht Paraphasie, wenn die Bahn $B A$ nur voriibergehend gesperrt oder wenn $A$ in Unordnung gerathen ist.

Auf Grund seines Schemas hat Lichtheim sieben einfache Krankbeitsbilder aufgestellt, die sich leicht ergeben, wenn man eine einzelne Bahn oder eins der beiden Centren als unterbrochen annimmt. Von den für diese sieben Krankheitsbilder eingeführten Namen sind die ron Wernicke gewählten am meisten iblich geworden, so dass auch wir uns derselben bedienen werden. Die durch Störungen in den Centren $M$ und $A$ herrorgerufenen werden als "corticale" bezeichnet, diejenigen der peripherisch davon gelegenen Bahnen als „subcorticale", die der zum Centrum $B$ führenden Bahnen als „transcorticale. Die Unterbrechung der Bahn $M A$ bedingt die „Leitungsaphasie".

Vergleicht man nun unseren Fall mit diesen Krankheitsbildern, so zeigt sich, dass keins derselben passt. Indessen hat Lich theim 
schon darauf hingewiesen, dass natiurlich auch zuweilen mehr als eine Bahn gestört sein könne, und erwähnt unter den so zum Vorschein kommenden Symptomencomplexen speciell ein eigenes Krankheitsbild, das entstehen müsste durch gleichzeitige Unterbrechung von $A M$ und $A a$. Vorhanden sein müssten die Symptome der corticalen sensorischen Aphasie, nur das Schriftverständniss müsste erhalten bleiben (das laute Lesen würde über $O A B M m$ erfolgen, statt für gewöhnlich über $O A M m$ ). Eine Mittheilung als Beleg für die Existenz dieses Symptomenbildes konnte er nicht auffinden. Auf den ersten Blick scheint es nun, als sei unser Fall geeignet, diese Liicke auszufüllen. Er wäre demnach zu bezeichnen als ein Fall von subcorticaler sensorischer Aphasie combinirt mit Leitungsaphasie.

Indessen wäre diese Auffassung zunächst doch nur eine oberflächliche. Die genaue Beobachtung des Kranken ergiebt Eigenthümlichkeiten in dem Bilde, sozusagen „Feinheiten" oder „individuelle Züge", an deren Erklärung das Li chtheim'sche Schema scheitert, weil es zu "grob" oder allzu "schematisch" ist. Es erklärt nicht, .weshalb bei dem Kranken die Paraphasie viel ausgeprägter war, als die Paragraphie. Lichtheim verfehlt nicht, besonders hervorzuheben - wie es auch nach seinem Schema sein muss, da die Schrift vollkommen von der Sprache abhängig ist - , dass die Störungen der Schriftsprache sich viel langsamer ausgleichen, als die der Lautsprache. Von den beiden Lichtheim'schen Wegen für das Schreiben wäre in unserem Falle natürlich nur der über $B M E$ in Betracht gekommen. Die Störung des Weges $M A$ hätte die Paragraphie an und für sich erklärt, unerklärt bleibt nur ihr Zurückbleiben gegeniiber der Paraphasie.

Diese für Lichtheim unerklärliche Unabhängigkeit der Schrift von der Sprache scheint allerdings sehr selten zu sein; denn man findet in der Literatur fast gar keine Angaben darïber. Ob indessen immer genügend hierauf geachtet wurde? K u ss maul sagt darüber „gewöhnlich leidet bei jeder Form von Dysphasie die Schriftsprache in noch weit höherem Maasse" und ferner ,beim Kranken verrathen sich die functionellen und organischen Läsionen des Gehirns noch leichter in der Schrift, als in der Rede". Einen Fall von Osborne, in dem hochgradige Paraphasie fast ohne Paragraphie vorhanden war, bezeichnet er als einzig in der Literatur. War der Unterschied zwischen Sprache und Schrift bei unserem Kranken auch nicht so überraschend wie dort, so war er doch immerhin auffallend genug, dass selbst seine Umgebung sich darïber wunderte.

Noch in einem anderen Punkt entspricht der Kranke nicht den 
Anforderungen Li chtheim's. Durch die angenommene Unterbrechung in der Bahn $M A$ müsste die willkürliche Innervation der Wortklangbilder gestört sein. Die so erzeugte Sprachstörung soll nun in frischen Fällen dadurch ausgezeichnet sein, dass der Wortschatz zwar sehr reich sei, dass aber die Sprache incorrect werde, die gebrauchten Wörter nicht selten verstümmelt, mitunter verkehrt gebraucht hervorkämen. Wenn diese Paraphasie jedoch rückgängig werde oder wenn sie - was für unseren Fall zutrifft - von vornhinein wenig hochgradig entwickelt sei, so trete eine Störung des Wortgedächtnisses, eine Amnesie deutlich hervor. Sie zeige sich gar nicht oder viel weniger in der fliessenden Rede, welche relativ selten stocke, viel hochgradiger hingegen, sobald dem Kranken aufgegeben werde, vorgezeigte Gegenstände zu benennen. Dabei zeige sich gewöhnlich, dass Namen, mit denen die Kranken in der fliessenden Rede operiren, ihnen absolut nicht einfallen, sobald man unter Vorzeigung des betreffenden Objects sie auffordere, diese Namen zu nennen. Von dieser Wortamnesie fand sich bei unserem Patienten keine Spur. Nach Wernicke fällt in leichten Fällen nicht so sehr das Verwechseln der Worte, als die subjectiv gefühlte Schwierigkeit auf, sie zu finden. Die Sprache ist stockend, mit langen Pausen, der Kran ke ringt bach dem Ausdruck. Auch hiervon zeigte unser Patient gar nichts; er plauderte gern, ganz harmlos in einem fort und in der grössten Gemiithlichkeit.

Auf dem ersten Anblick lässt sich uuser Fall also nach dem Lichtheim'schen Schema anscheinend leicht deuten, ja er scheint sogar einem Krankheitsbilde zu entsprechen, dessen Vorkommen Lichtheim theoretisch fordert; aie genauere Betrachtung ergiebt jedoch Einzelheiten, die mit demselben in Widerspruch stehen.

Noch unhaltbarer wird derselbe, wenn wir die von Wernicke entwickelten, speciell das Lesen und Schreiben betreffenden Anschauungen zu Grunde legen. Lesen und Schreiben geschieht nach ihm nur nit Hülfe des oben definirten „Wortbegriffs". Beim Lesen innervirt das Schriftbild O den Wortbegriff; das Schreiben geschieht so, dass der Wortbegriff das zugehörige Schriftbild innervirt, und dass dieses nun nachgezeichnet wird. Dies soll sich für jeden einzehnen Buchstaben wiederholen, da wir nach Grashey nur buchstabirend lesen und schreiben können. Erst wenn alle einzelnen Buchstaben gelesen sind, entsteht der ganze Wortbegriff. Zum Verständniss des Gelesenen sowie zum willkürlichen Schreiben ist die Unversehrtheit der Verbindung zwischen Wortbegriff und Begriffscentrum nöthig. Um zu bezeichnen, dass eine Störung der Schriftsprache durch eine Läsion 
des Wortbegriffs $(A+A M+M)$ an irgend einer Stelle bedingt ist, wird die Störung als eine "verbale" bezeichnet.

Mit diesen Lehren verträgt sich die von uns für unseren Fall gemachte Annahme einer Leitungsaphasie nicht; denn Leitungsaphasie, sagt Wernicke kurz und bundig, bedingt vollkommene Alexie, während unser Patient sehr gut lesen konnte. Nun finden sich bei $W$ ernicke noch folgende Bemerkungen ïber Schreib- und Lesestörungen : Wo glatt und mit vollständig erhaltener Buchstabenform geschrieben werden kann, nur die Bucbstaben untereinander verwechselt und die Wörter so entstellt werden, da handelt es sich gar nicht um Agraphie und Paragraphie, sondern um das Symptom der Paraphasie, das auch beim Schreiben zum Ausdruck kommt, um geschriebene Paraphasie mit einem Worte. Derartiges könnte vorkommen bei transcorticaler sensorischer Aphasie. Ferner sagt er vom Lesen: wo noch gelesen werden kann, nur aber beim Lautlesen die Paraphasie ebenso wie beim Sprechen sich geltend macht, muss eine andere Form der Aphasie als Leitungsaphasie vorliegen. Er vermuthet, dass an einem solchen Falle eine amnestische oder eine in Ausgleichung begriffene corticale oder transcorticale sensorische Aphasie betheiligt sei.

Es ist klar, dass die in unserem Fall beobachtete Schreib- und Lesestörung den hier geschilderten Bildern entspricht. Indessen seine. Zurückfuihrung auf corticale sensorische Aphasie ist unzulässig, da hierbei der zum Lesen und Schreiben erforderliche Wortbegriff ebenso gestört ist, wie bei der Leitungsaphasie, und es bliebe somit nur noch die Annahme einer transcorticalen sensorischen Aphasie tibrig. Die Paraphasie mit erhaltenen richtigen Buchstabenformen (geschriebene Paraphasie) würde sich dadurch deuten lassen. Aber da es eben geschriebene Paraphasie ist, fragt man vergebens, warum war sie weniger ausgeprägt als die gesprochene? Wernicke sagt sogar, die Paraphasie ist stärker beim Schreiben als beim Sprechen; bei transcorticaler sensorischer Aphasie muss ein sehr frappanter Gegensatz zwischen der nur geringen Paraphasie beim Sprechen und der hochgradigen beim Schreiben vorbanden sein. Ganz unerklärt bliebe aber das Verständniss für das Gelesene. Von irgend einer in Ausgleichung begriffenen Form von Aphasie kann vollends gar keine Rede sein darauf wird ja oft alles das in bequemer Weise zurückgeführt, was in das Schema nicht passen will - denn das Krankheitsbild, welches von Beginn an ärztlich beobachtet werden konnte, hat überhaupt keine Veränderungen gezeigt.

Das Wernicke'sche Schema versagt also bei der Erklärung unseres Falles und muss versagen; denn für dieses Schema ist charak- 
teristisch, dass die Lautsprache die Schriftsprache völlig beherrscht; die Störungen jener müssen in vergrössertem Maassstabe sich in den Störungen dieser wiederspiegeln, während bei unserem Kranken diese Abhängigkeit fehlte. Dagegen hätte sich wenigstens sein Verhalten beim Lesen nach den Lichtheim'schen Anschaungen sehr gut deuten lassen. Der gewöhnliche Weg tiber $O A M m$ war versperrt. Der Kranke liest also iuber $O A B M m$, ein Vorgang, der natiurlich langsamer von Statten geht als auf dem eingeübten alten Wege. Daher das bedächtige Lesen. Er macht dabei keine Sprachfehler; denn das nach Lichtheim für die Correctheit der Aussprache erforderliche Auftauchen der Klangbilder und deren Treten uber die Schwelle des Bewusstseins findet ja statt. Die beim Lesen vorhandenen sehr spärlichen Fehler könnte man sehr wobl als Versprechen auffassen, wie es ja auch dem Gesunden beim Vorlesen passirt.

Die vorstehende ausfïhrliche Analyse zeigt, dass selbst ein so einfacher und uncomplicirter Fall wie der unserige - und gerade deshalb wurde eine so ausfuthrliche Analyse gegeben - einem Verständniss auf Grund der Lichtheim'schen und Wernicke'schen Anschauungen nicht ohne Weiteres zugänglich ist. Ganz unverständlich ist er für die Anschauungen Wernicke's, anscheinend verständlich für die Lichtheim's, selbst psychologische Feinheiten der genauen Beobachtung kann man zum Theil danach verstehen (geringere Paraphasie beim Lesen als beim Sprechen, das bedächtige Lesen), andere aber, vor Allem der Gegensatz zwischen der unbedeutenden Paragraphie und der stärkeren Paraphasie, kann auch das Lichtheim'sche Schema nicht erklären; die von ihm verlangte Wortamnesie beim Benennen von Gegenständen fehlt. Sollen wir deshalb den Fall als zur Zeit unverständlich oder dem Schema zur Liebe als Ausnahme hinstellen? Bei einem klinisch so einfachen Fall dürfte das nicht angebracht sein. Wernicke ${ }^{1}$ ) sagt mit Recht, es werde mit dem Gehirn ein vergebliches Bemühen sein, den wahren $\mathrm{Zu}$ sammenhang der Erscheinungen zu ermitteln, solange man sich nicht den Weg zum Verständniss des einzelnen Falles gebahnt habe. Man mïsse den einzelnen Fall in seiner individuellen Schattirung versteben und den zahlreichen Complicationen, die die Natur meist biete, mit Bewusstsein gegenüber stehen.

Den Weg za einem Verständniss zeigt das Schema von Kussmaul, in dem die Schriftsprache selbständiger dasteht. Noch viel unabhängiger von der Lautsprache ist sie, wenn man sich den An-

1) Lehrbuch der Gehirnkrankheiten. Bd. II. S. 10. 
schauungen $\mathrm{Cb}$ arcot's und seiner Schüler (Ballet u. A) über die innerliche Sprache anschliesst. Als ganz besonders einseitig ist mir immer die Annahme Wernicke's erschienen, dass das Schreiben und Lesen nur buchstabirend geschehe. Hierzu ist er gekommen, indem er die bekannte, viel citirte Beobachtung von Grashey als allgemein guiltig betrachtete. Seitdem gilt diese Lehre in Deutschland als eine Art von Dogma. Beweise von deren Allgemeingiultigkeit fehlen aber durchaus. Zweifellos ist bei schriftungewandten Leuten der Vorgang ein derartiger, allein warum andere Menschen, die anhaltend mit der Schriftsprache zu thun haben, nicht allmählich lernen sollten, die Wörter oder wenigstens die grosse Mehrzahl der gebräuchlichsten Wörter als Ganzes aufzufassen, ist gar nicht einzusehen. Wernicke selbst lässt das z. B. beim Schreiben der Namensunterschrift zu. Wenn es aber hierfür zulässig ist, muss es meines Erachtens auch für andere Wörter zulässig sein; denn es giebt deren eine Menge, welche dem, der viel zu lesen und zu schreiben hat, bei Weitem häufiger vor Augen kommen, als der eigene Name. Uebrigens ist die Wernicke'sebe Lehre auch durch directe Zeitbestimmungen entscheidend widerlegt, die ergaben, dass ein einzelner Buchstabe ungefähr gleich lange Zeit braucbt, um erkannt $\mathrm{zu}$ werden wie ein kurzes Wort. Ja in den Versuchen von Titchener dauerte die Erkennung eines kurzen Wortes sogar noch etwas weniger lange, als die eines einzelnen Buchstabens. Daraus schliesst auch $W u n d^{1}{ }^{1}$, dass wir beim Lesen, sobald wir nur über die Anfänge des Lesenlernens hinaus sind, nicht das Wort in seine Buchstaben trennen, sondern es als in Einem Act gegebenes Ganzes auffassen. Unser Patient hatte nun als Kaufmann auf seinem Comptoir sich täglich Stunden lang mit Lesen und Schreiben zu beschäftigen, und ich glaube daher annehmen zu können, dass er sich für die meisten Wörter das buchstabirende Lesen und Scbreiben abgewöhnt hatte. Sieht man in Bezug auf diesen Punkt von den Wernicke'schen Anschaungen $\mathrm{ab}$, so lässt sich der Fall auf Grund seines Schemas völlig befriedigend deuten.

Das Sprechen erfolgte über $B M$ unter Controle der Bahn $B A M$. Deren Unterbrechung in $A M$ bedingte die Paraphasie. Das laute Lesen erfolgte statt gewöhnlich über $O A M$ über $O A B M$, daher der etwas langsame und bedächtige Charakter desselben. Da so die Klangbilder mitwirken konnten, fehlte hierbei die Paraphasie oder war wenigstens nicht stärker, als das Versprechen eines Gesunden beim lauten Lesen. Letzterer corrigirt den gemachten Febler, da er

1) Vorlesungen über die Menschen- and Thierseele. 2. Aufl. S. 303. 
ihn selbst hört. Unser Patient konnte dies nicht, da er sprachtaub war und selbst nicht hörte, was er vorlas. Das Schreiben in gesunden Tagen erfolgte über $B M O E$ unter Controle der Bahn $B A M$ (also unter Benutzung der Bahn für die Sprache). Die infolge von Unterbrechung der Bahn $A M$ za erwartende Paragraphie oder, wie man in unserem Falle besser sagen wird ,gesehriebene Paraphasie", wurde durch das Eintreten der Bahn $A O$ ausgeglichen. Auf die allmähliche Einübung dieser Bahn könnte man es sogar zurückführen, dass das Zurïckbleiben der Paragraphie hinter der Paraphasie besonders hervortrat, nachdem die Krankheit schon einige Wochen bestanden hatte.

Somit können wir an der Diagnose einer Leitungsaphasie für die Erklärung der Sprach- und Schreibstörungen festhalten. Das von Wernicke ${ }^{1}$ ) neuerdings angegebene und in einem Falle beobachtete positive Kennzeichen derselben, dass das Nachsprechen der Wörter in auffallender Weise gelitten hat, konnte wegen der Sprachtaubheit nicht zur Beobachtung gelangen. Störungen des Gedächtnisses und der Aufmerksamkeit können in unserem Falle, da sie völlig fehlten, zur Erklärung nicht herangezogen werden; er muss sich durchaus auf Leitungs- oder Associationsstörungen zuriickfübren lassen. Dass dies für einen Theil derselben durch Annabme einer Leitungsaphasie möglich ist, wurde im Vorhergehenden gezeigt. Dabei ergab sich zugleich die Nothwendigkeit des Erhaltenseins der Wortklangbilder; mitunter war ihre Mitwirkung, mitunter ihr Ausfallen zur Erklärung des Beobachteten nöthig. Dies konnte in zureichender Weise geschehen, ohne dass als gezwungen erscheinende Annahmen nöthig waren. In dem Folgenden bleibt nun noch zu zeigen übrig, dass die Worttaubheit nur als subcorticale sensorische Aphasie aufgefasst werden kann und somit auf eine Störung der Bahn $A a$ bezogen werden muss.

Beide Bahnen, deren Störung zu einem Verständniss unseres Falles nöthig ist, münden in $A$ ein. Es steht der Annahme nichts entgegen, dass sie kurz vor ihrer Einmündung einander dicht benachbart sind. Sie konnten hier also leicht durch einen kleinen Blutungsherd eine Diagnose, welche wir nach den klinischen Erscheinungen stellen müssten, gleichzeitig getroffen werden, so dass auch hier Theorie und Beobachtung gut $\mathrm{zu}$ einander passen.

\section{Das klinische Bild der subcorticalen sensorischen Aphasie und seine Deatung.}

Das klinische Bild der subcorticalen sensorischen Aphasie ist nach den Angaben Li ch th e i m's, seines Entdeckers, scheinbar ausser-

1) Gesammelte Aufsätze. \$. 324 . 
ordentlich einfach: verloren gehen nur das Sprachverständniss, die Fähigkeit nachzusprechen und auf Dictat zu schreiben. Doch ergeben sich aus der genauen Beschreibung, die er von einem solchen Falle macht, noch einige wichtige Einzelheiten, auf die wir eingehen mïssen. Sein Patient machte völlig den Eindruck eines Stocktauben. Man kann nur schriftlich mit ihm verkehren, indem man auch die geringste Kleinigkeit aufschreiben muss. Auch seine Frau versteht er so gut wie nicht, nur einige wenige Worte liest er ibr sehr unvollkommen von den Lippen ab. Lichtheim würde ibn wahrscheinlich fur vollkommen taub gehalten haben, wenn ibm nicht die Frau versichert hätte, dass er sehr fein höre, sehr empfindlich gegen jedes Geräusch sei und behaupte, es thue ihm weh. Auffallend war jedoch für Lichtheim immer seine geringe Aufmerksamkeit für Geräusche, welche besonders leicht den Eindruck, dass er taub sei, hervorruft. Dieselbe geringe Aufmerksamkeit zeigt sich auch, wenn man mit ihm redet. Er hat nicht das Bestreben anderer Sprachtauber, das, was man ihnen sagt, zu verstehen, sondern achtet gar nicht darauf; man muss ihn erst dadurch, dass man ihn anstösst, zur Aufmerksamkeit zwingen. Er hört es, wenn man pfeift und singt, erkennt jedoch die Melodie nicht. Wenn seine Kinder im Zimmer vierstimmig singen, was er früher sehr gern hatte, befiehlt er ihnen aufzuhören und sagt, sie schreien zu stark. Als Lichtheim ihm vorpfeift, „Hörst Du mein Vaterland", sagt er: "Noch einmal, vielleicht erkenn ich es", er erkennt es aber nicht. Er kann absolut nicht nachsprechen, wenn man ihn schriftlich dazu auffordert. Lichtheim spricht ihm vor, „ich heisse". Er fixirt die Lippen und bringt endlich heraus "Isch", sonst gelingt ihm nichts. Schriftlich aufgefordert, auf Dictat zu schreiben, sagte er, „aber ich höre es nicht, das ist eine dumme Geschichte mit dem Gebör". Als Lichtheim trotzdem fortfährt zu dictiren, sagte er, "das ist eine Kunst dictiren, wenn man nicht hört".

Lichtheim macht nun die Bemerkung, die anderen Fälle von Sprachtaubheit, die er gesehen habe, hätten sich ganz wesentlich von diesem unterschieden. Bei ihnen habe man eher Gefahr gelaufen, die Sprachtaubheit zu übersehen; die Kranken beantworteten alle Fragen, die man an sie richtete, nur passte die Antwort nicht auf die Frage, und man lief viel eher Gefahr, die Kranken für verwirrt als für taub zu halten. Ganz anders dieser Kranke: er schenkte den Fragen, die man an ihn richtete, gar keine Aufmerksamkeit, beantwortete sie niemals und machte gerade deshalb den Eindruck eines Tauben. Endlich sei im Gegensatz zu den meisten übrigen Fällen von Sprachtaubheit eine Eigenthümlichkeit die vollkommene Persistenz dieses Sym- 
ptoms. Lichtheim glaubt, dass diese Absonderlichkeiten diagnostisch verwerthbare Eigenthümlichkeiten des Symptomencomplexes der subcorticalen sensorischen Aphasie darstellen.

Ein Vergleich des Lichtheim'schen Falles mit dem unserigen zeigt sofort die völlige Uebereinstimmung zwischen beiden: Dieselben Symptome und dasselbe psychologische Verhalten des Kranken. Da in beiden Fällen Beobachtete lässt sich in vier Punkte kurz zusammenfassen: 1. der völlige Verlust des Verständnisses für die Sprache und die Musik trotz erhaltenen Hörvermögens für Töne und Geräusche; 2. die Unmöglichkeit, auf Dictat zu schreiben; 3. die Unmöglichkeit, Wörter nachzusprechen trotz erhaltener Fähigkeit, sprachliche Laute nachzumachen (letzteres in unserem Falle, Lich th eim giebt nichts darüber an), ebenso trotz der Möglichkeit Töne nachzuahmen, Unfähigkeit Melodien nachzumachen; 4. die völlige Unveränderlichkeit des Krankheitsbildes.

Der Anschein in beiden Fällen, als ob man Taube vor sich habe, wurde durch die genauere Untersuchung nicht bestätigt. Hätten diese Kranken infolge ihrer Gehirnerkrankung ïberhaupt nicht mehr gehört, d. h. hätten sie nicht nur Worte nicht gehört, sondern überhaupt Mangel an Hörvermögen aufgewiesen, so hätte man es nach der Bezeichnungsweise von $\mathrm{Munk}$ mit Hirn- oder Rindentaubheit zu thun. Diese liegt nicht vor, denn beide Kranke hörten gut. Auch Seelentaubheit liegt bestimmt nicht vor. Dies geht besonders aus der Untersuchung unseres eigenen Patienten hervor. Ein Seelentauber mïsste wohl Geräusche und Töne hören, dürfte aber nicht im Stande sein, deren Bedeutung aufzufassen. Hiervon ist bei unseren Kranken keine Rede. Er deutet jedes Geräusch, jeden Ton völlig richtig, ja er unterscheidet das Geräusch eines bestimmten Wagens (den seines Arztes) von dem Geräusch anderer Wagen ganz genau u. s.w. Was bei ihm vorhanden ist, ist einzig und allein die "Worttaubheit".

Von der Worttaubheit giebt Ballet ${ }^{1}$ ) folgende Definition: „Ein von Worttaubheit betroffenes Wesen wird die Klänge hören, wird sie auf den Gegenstand zu beziehen vermögen, der sie hervorbringt, wird aber nicht den Sinn der gesprochenen Worte erfassen“. „Das Wort wird also als Klang vernommen, aber als nichtssagender Klang, der unfähig ist, eine Vorstellung hervorzurufen. Er beeinflusst das Hirn so, als ob es ihn zum ersten Male hörte, was so viel sagen will, als dass die graue Substanz keine Erinnerung, kein Gebörsbild dieses Wortes mehr besitzt. Die Worttaubheit rührt also her von einem

1) Die innerliche Sprache. Deutsche Uebers. S. 87 u. 94. 
Verlöschen der Gehörsvorstellungen, und zwar von einem derartigen Erlöschen, dass nicht nur das Hervorrufen ohne die sie bedingende Sinneswahrnehmung unmöglich ist wie in der Amnesie, sondern dass anch die Sinneswahrnehmung selbst, die vor Kurzem das Bild entstehen liess, nicht mehr fähig ist, dieses Bild wieder zu erwecken". „Die Worttaubheit, sagt ferner Bernard"), berubt in der Unmöglichkeit, die Bedeutung des gehörten Wortes zu begreifen und selbst aller Klänge, die hergebrachtermaassen Träger von Vorstellungen geworden sind, d. i. also Verlust oder mehr oder weniger vollständige Störung des Gedächtnisses für die Bedeutung der Laute."

Bei der Worttaubheit infolge von subcorticaler sensorischer Aphasie liegt nun meines Erachtens etwas ganz anderes vor. Solche Kranke hören iiberhaupt keine Worte, sie haben nicht die Empfindung, dass in Worten zu ihnen gesprochen wird, sie hören die Worte nicht als Worte, die nur keinen Sinn für sie haben, sie hören Worte als vollkommene Geräusche. Wie die Wörter physikalisch nichts sind, als ein Complex von Tönen und Geräusehen, so und nicht anders kommen sie diesem Kranken zum Bewusstsein. Die Complexe von Tönen und Geräuschen, die sie hören, werden nicht so miteinander versehmolzen, dass sie die Vorstellung baben, ,es ist ein Wort, das ich gehört habe oder es könnte vielleicht eins sein". Sie sind also nicht blos "Worttaube, sondern sie sind, was man streng davon trennen sollte, vollkommen „Sprachtaube“. Sie sind, wenn man will, seelentaub für . Worte, sie verstehen nicht nur nicht den Sinn der Worte, sondern nicht einmal die Bedeutung dessen, was in ihr Ohr gedrungen ist, als eines Wortes. Sie haben nicht die Empfindung, dass ein Mensch spricht, sondern die, als ob er etwas beliebiges sinnioses vor sich hin summt, vielleicht die Empfindung, die Jemand hat, der mitten in einem Saal voll von durcheinander sprechenden Menschen steht und auch nur ein Gewoge und Gesumme von Stimmen, aber keine Worte hört. Unser Kranker sagte, „es ist Wirrwarr", „Alles geht mir durcheinander", „es ist ein wirres Gewoge fn meinem Ohr". Psychologisch ausgedrückt, die Gehörsempfindungen sind nicht mehr im Stande, blosse Wortvorstellungen zu erregen, geschweige denn Wortvorstellungen mit dem ihnen zukommenden Sinn.

Damit erklärt sich auch psychologisch das Verbalten eines solchen Sprachtauben sens. strict. gegenüber dem eines Worttauben. Jener hat nicht mehr die Empfindung, dass Jemand ihn mit Worten anredet, folglich bört er auch gar nicht hin, ebensowenig wie ein Ge-

1) De l'aphasie. 2. Édit. p. 133. 
sunder hinhört, wenn ein Anderer in seiner Nähe Beliebiges vor sich hinsummt, sondern ruhig sich mit seinen eigenen Gedanken beschäftigt; denn auch der Gesunde beachtet für gewöhnlich die Geräusche in seiner Umgebung nicht, sondern nur, wenn er ihnen aus irgend einem Grunde seine Aufmerksamkeit zuwendet. Andere Worttaube (cortical oder transcortical sensorisch Aphasische) haben wirkliche Wortempfindungen, nur verkennen sie den Sinn derselben. Daher deren abweichendes Verhalten beim Anreden; sie hören zu, sie glauben verstanden $z u$ haben, sie antworten daher auch, aber Mangels richtigen Wortverständnisses sinnlos.

Wir verstehen nun auch, weshalb ein solcher Kranker einfache Buchstaben erkennt und sogar nachsprechen kann; denn die Buchstaben sind ja nichts anderes als Töne und Geräusche. Deshalb müssen auch die Consonanten stumm gesprochen werden, soll er sie versteben. Er macht sie nach, wie er andere Geräusche nachmacht. Uebrigens taucht bei den Buchstabengeräuschen die Vorstellung des zugehörigen Buchstabens auf, ebenso, wie bei anderen Geräuschen die Vorstellung ihrer Quelle auftaucht; denn auf Verlangen schreibt er den gehörten Buchstaben auch hin. Die Wörter dagegen vernimmt er als complicirte Geräusche, die er so wenig nachmachen kann wie ein Gesunder, z. B. das Fahren eines Wagens auf der Strasse u. s. w.

Für beide Kranke ergiebt sich das merkwürdige Verhältniss, dass einfache Töne (Stimmgabel) oder musikalische Klänge (Töne von Instrumenten) den Buchstaben vollkommen entsprechen und sogar nach der Klangfarbe auf das specifische Instrument, von dem sie herrühren, bezogen werden, wie unter Umständen Wortgeräusche auf bestimmte dem Kranken bekannte Personen, die er an dem Timbre ihrer Sprache erkennt, dass dagegen Melodien sich verhalten wie Wörter oder Worte als Reihen von Wörtern. Der Sprache entspricht die Musik vollkommen auch bezüglich des Nachmachens. Beide Kranke waren nicht nur sprach-, sondern auch musiktaub.

Dass diese Kranken aber nicht worttaub sind, weil sie, wie die gewöhnliche Definition der Worttaubheit lautet, die Wortklangbilder verloren haben, zeigt sich daraus, dass letztere beim Sprechen, Lesen und Schreiben bei ihnen mitwirken; sie müssen also noch vorhanden sein. Dementsprechend gab unser Kranker auch eine verlangte Melodie richtig an, innerlich fehlte sie ihm so wenig wie die Wortklangbilder. $\mathrm{Ob}$ er eine Melodie nach den Noten hätte erkennen, resp. einen gehörten Ton als Note bätte niederschreiben können, kann ich nicht angeben, da er Notenschrift nicht verstand.

Demnach ist für das vorliegende Krankheitsbild charakteristisch, 
dass, während alle übrigen Gehörsempfindungen, auch die von Buchstaben und Tönen, intact sind und die entsprechenden richtigen Gehörsvorstellungen erzeugen, nur die durch die Sprache und die Musik von aussen hervorgerufenen akustischen Empfindungen nicht mehr die zugehörigen Vorstellungen von Wortklangbildern und Melodien hervorrufen und natürlich erst recht nicht die Vorstellungen der Bedeutung der einzelnen Wortklangbilder, während central und associativ die sensorisch akustischen Erinnerungsbilder von Wörtern und Melodien erweckt werden können, oder mit Einem Wort ausgefallen sind einzig und allein die Wahrnehmungsvorstellungen von Wörtern und Melodien.

Um zu einem weiteren Verständniss der Eigenthümlichkeiten des Krankheitsbildes zu kommen, ist daran zu erinnern, dass sämmtliche Gehörswahrnehmungen, die wir haben, bereits zusammengesetzte psychologische Vorgänge sind. Schon die Töne (Klänge) bestehen bekanntlich stets aus einem Haupt- und zahlreichen Nebentönen. Sie sind also die Summe einer Anzahl einfacher Empfindungen, die, da wir gewöhnt sind, sie in festen Verbindungen zu hören, für uns zu einheitlichen Vorstellungen verschmolzen sind. Da nun zwischen Klang und Geräusch nur graduelle Unterschiede bestehen, die durch die zahlreichen dissonanten Bestandtheile und die massenhaft entstehenden Schwebungen bei den Geräuschen bedingt sind, so ist das Hören der Geräusche ein ähnlicher gewohnheitsgemässer Verschmelzungsprocess. Nichts anderes als Klänge und Geräusche sind aber die Vocale und Consonanten unserer Sprache, so dass es verständlich ist, wenn Kranke gegenüber den Sprachelementen und anderen Schallquellen das gleiche Verhalten zeigen. Ein Unterschied tritt erst hervor, wenn das die Gehörsempfindung auslösende Moment einerseits aus einer zusammengesetzten Reihe von einzelnen Geräuschen und andererseits aus einer charakteristischen Reihe von Buchstabengeräuschen oder Tönen, d. h. von Wörtern oder Melodien besteht, also aus Elementen, die Träger von sprachlichen oder musikalischen Vorstellungen geworden sind; denn eine Aufeinanderfolge von Einzelgeräuschen, wie sie z. B. das Fahren eines bestimmten Wagens, die Stimme von Thieren $u$. dergl. erzeugt, wird durchaus richtig erkannt, nicht aber die einfachste Folge von zu einer Melodie verbundenen Tönen oder von selbst nur wenigen Buchstaben, die ein Wort bilden. Mit Ruicksicht auf ersteres kann man daher nicht sagen, dass psychologisch etwa das successive Associationsvermögen gestört gewesen sei. Vielmehr verhielten sich die beiden Reihen von Empfindungscomplexen psychologisch so grundverschieden, dass man nothwendig auf den 
Gedanken kommen muss, die Verschiedenheit rühre davon her, dass dabei verschiedene physiologische Substrate mitwirken; denn man wird sich kaum eine Idee davon machen können, wie in demselben nervösen Substrat zwar die eine Art von Gehörswahrnehmungen, nicht aber die andere, davon nur graduell verschieden ausgelöst werden könnte. Die Schwierigkeit ist um so grösser, als letztere nur peripher als Wahrnehmungsvorstellung nicht hervorgerufen werden kann, wohl aber noch central direct oder associativ als Erinnerungsvorstellung.

Nach Bastian soll es bekanntlich drei Grade von Erregbarkeitsverminderung eines Centrums geben. Für den leichtesten ist charakteristisch, dass das Centrum noch durch Association von einem anderen Centrum und durch directen peripherischen Reiz erregbar ist, aber nicht mehr durch den Willen. Bei der folgenden Stufe wirkt nur noch der directe sensible Reiz, und beim höchsten Grad auch dieser nicht mehr. Es müsste also bei unserem Krankheitsbild, wenn man nur eine nicbt weiter differenzirte Hörsphäre annimmt, hierin geradezu, eine Umkehr, und zwar nur für sprachliche und musikalische Reize stattfinden, ein Vorgang, der sich jeder Erklärung entzieht.

Somit kommen wir zn demselben Satz wie schon früher Kus smaul ${ }^{1}$ ), nur in Bezug auf die Musik entsprechend erweitert: „Die Wahrnehmung von Klängen und Geräuschen, die für sich als Vocale und Consonanten begriffen werden, und ihre Fügung zum akustischen Wortbild, das als Symbol dieser oder jener Vorstellung erfasst wird, sind verschiedene Functionen, die an verschiedene Centraltheile gebunden sind." Die subcorticale sensorische Aphasie ist dasjenige Krankheitsbild, bei dem dies in den Symptomen klar zu Tage tritt. Preyer ${ }^{2}$ ) sondert in seinem Schema von den Sprachvorgängen das Schallcentrum sogar in ein Laut-, Silben- und Wortcentrum. Aus der Beobachtung unseres Falles würde sich zunächst nur die Nothwendigkeit einer Abtrennung des sensorischen Silben- und Wortcentrums elgeben, während das Lautcentrum von dem allgemeinen akustischen Centrum nicht gesondert zu werden braucht.

Eine derartige Trennung würde nun mit der Entwicklungsgeschichte der Sprache beim Kinde gut in Uebereinstimmung sein; denn in der That geht dem Sprechen und Hören der Wörter das Sprechen und Hören der Buchstabenlaute voraus, worin ich Preyer nach Beobachtungen an meinen eigenen Kindern durchaus beistimmen

1) Die Störungen der Sprache. 2. Aufl. S. 175.

2) Die Seele des Kindes. 2. Aufl. Fig. 2, 3 u. 4, S. 236 u. flg. 
muss. Silben- und Wörter spricht und versteht das Kind erst, nachdem es lange vorher die Laute sich schon vorgesprochen hat, und theils dadurch, theils durch den Einfluss der Umgebung in den Besitz der zugehörigen sensorischen Erinnerungsbilder gekommen ist.

Nimmt man nun an, dass die simultane Association versehiedener Gehörsempfindungen oder deren Verschmelzung zu der Vorstellung eines Lautes resp. eines Tones ihr physiologisches Correlat in der centralen Verknüpfung entsprechend verschiedener nervöser, empfindender Elemente findet, so würde die weitere Annahme gestattet sein, dass ein solcher Complex erregend wirkt auf ein anderes durch Leitungsbahnen mit ihm verbundenes Element (oder eine Anzahl von solehen), dessen Erregung somit aus der Summe jener Erregungen resultirt. Der Verknüpfung der letzteren würde die successive Association der Laute zu Wort- resp. der Töne zu Musikvorstellungen entsprechen, und erst aus der Association dieser fertigen Vorstellungen mit anderen würde das Verständniss für die Bedeutung des gehörten Wortes bervorgehen. Die Probe auf die Unversehrtheit des einen oder des anderen sensorisch-akustischen Associationsvorganges bildet die Möglichkeit des Nachsprechens vorgesagter Wörter oder Buchstabenlaute, des Nachsingens von Tönen oder Melodien. Es wïrde somit das Wortklangcentrum dem Lautklangcentrum übergeordnet sein, und man würde durchaus begreifen, warum beim Kinde jenes erst successive in Function treten kann, nachdem dieses entsprechend eingeübt ist. Später freilich geht das Gefühl für die Combination der Wortklangbilder aus Lauten dem Kinde verloren, indem die Erinnerungsbilder der letzteren im Bewusstsein zurücktreten, und klar nur noch die Erinnerungsbilder der Wortklangbilder als verschmolzene Einheiten infolge des Einflusses der Umgebung und der Uebung auftauchen. So gilt auch wohl hier bei der Sprache das paradoxe Resultat ${ }^{1}$ ), dass eine Bedingung des Gedächtnisses das Vergessen ist. Ohne das vollständige Vergessen einer ausserordentlich grossen Anzahl und das momentane Vergessen einer grossen Anzahl von Bewusstseinszuständen könnten wir uns überhaupt an nichts erinnern. Die Lautklangbilder aber werden vollkommen überflüssig, wenn die Wortklangbilder entstehen.

Später werden die vergessenen Buchstabenlaute dem Kinde beim Erlernen der Schriftsprache künstlich wieder beigebracht, und alsdann lernt es wieder aus einem Wortklang die Laute heraushören, ähnlich wie ein Musiker einen Accord in seine Töne auflöst, während ein nicht musikalisch Gebildeter das ebenso wenig vermag, wie Jemand, der

1) Ribot, Das Gedăchtniss. Deutsche Uebersetzung. S. 36 . 
nicht lesen und schreiben gelernt hat, ein Wort in seine Lautelemente zerlegen kanu. Ribot sagt ganz richtig: „Wir wissen lange Zeit nicht, dass die Worte einfachere Elemente voraussetzen; Viele wissen es nie".

Aus der bisherigen Darstellung geht wohl schon hervor, dass wir nicht auf dem Boden der Munk'schen Anschauung stehen, welche Rindengebiete, in denen die Sinnesempfindung steht, von solchen trennt, in denen die Empfindungen zu Vorstellungen erhoben werden. Daraus ist bekanntlich die Lebre entstanden, jede Vorstellung sei in eine bestimmte Nervenzelle des Gehirns deponirt, so dass die Erregung dieser Zelle die schlummernde Vorstellung wachruft. Nach dieser Annahme müsste in der Hörsphäre jedes Wortklangbild an einer Zelle haften, in die es sich beim erstmaligen oder öfteren Hören „eingegraben" hat, so dass die Hörsphäre mit fertigen, dort ein für alle Mal deponirten, bald über, bald unter der Schwelle des Bewusstseins stehenden Klangbildern bevölkert wäre. Die Bildung der sensorischen Sprachvorstellungen ist vielmehr ein äusserst zusammengesetzter Vorgang, der nur durch die Mitwirkung vieler verschiedener physiologischer Elemente zu Stande kommen kann. Die Wahrnebmungsund die Erinnerungsvorstellungen aber haften an denselben Elementen, von denen man jedoch annehmen muss, dass sie nicht mit solchen der allgemeinen Hörsphäre identisch, sondern dass sie Elemente einer besonderen Worthörsphäre sind. Zur Perception, wenn die letztere ausfällt, kommen die Wörter allerdings auch in jener, doch nur als unerkanntes Geräusch nicht als etwas, was Sprachcharakter hat. Dieses ist wohl $\mathrm{zu}$ unterscheiden von der obigen Munk'schen Lehre. Obschon Sprache und Musik sich hier als theoretisch ganz identisch zu erklärende Processe ergeben haben, müssen beide doch auseinander gehalten werden, da, wie die später folgende Casuistik ${ }^{1}$ ) lehrt, die pathologischen Störungen beider zuweilen unabhängig von einander sind. Die Centralstationen beider sind einander offenbar dicht benachbart.

Es ergiebt sich somit, dass die durch die klinische Beobachtung bei der subcorticalen sensorischen Aphasie symptomatisch gegebene Abtrennung eines Vermögens für das Hören der Wörter und Melodien vom übrigen Hörvermögen mit psychophysiologischen und der Entwicklungsgeschichte der Sprache entnommenen Anschauungen in Einklang zu bringen ist; es fragt sich nun noch, ob diese Lehre in anatomischen Befunden am Gehirn eine Stütze findet, so dass sie auch einer ana-

1) S. unten Fall 7. 
tomischen resp. anatomisch-physiologischen Deutung fähig ist. Infolge der noch mangelhaften Kenntniss von dem centralen Verlauf des Acusticus sind hier einige Luicken vorhanden, die vorerst noch durch hypothetische Annahmen ergänzt werden müssen.

Als centrale Endapparate des Acusticus sind die Ganglienzellen in der Rinde der beiden Schläfelappen zu betrachten; denn die klinische Beobachtung lehrt, z. B. der Fall von Wernicke und Friedländer'), dass deren Zerstörung vollständige Taubheit verursacht. Wahrscheinlich ist beim Menschen jeder Acusticus mit beiden Hemisphären in Verbindung; denn eine einseitige Rindenerkrankung scheint keine Taubheit des gekreuzten Ohres (wie beim Hunde) hervorzurufen. Wie der Acusticus in den Schläfelappen gelangt, wo die Kreuzung stattfindet, ob vielleicht vorher noch eine Unterbrechung der Babn durch Ganglien stattfindet, ist gänzlich unbekannt. Zwischen beiden Schläfelappen wird man eine Verbindungsbahn voraussetzen mïssen, die das Zustandekommen einer einheitlichen Wahrnehmung. von dem mit beiden Ohren gehörten vermittelt. Der vordere Theil beider Schläfelappen scheint seine Verbindung in der vorderen Commissur zu haben, während Fasern des Tapetum vorwiegend die hinteren Abschnitte beider Schläfelappen und insbesondere die lateralen Partien, die 3. bis 1. Schläfewindung, mit einander verbinden. ${ }^{2}$ ) Ganz sicher aber ist, dass das Sprachverständniss an die Unversehrtheit eines umschriebenen Theiles des linken Schläfelappen, der hinteren Partien der 1. und 2. Schläfewindung, gebunden ist. Da jedes $\mathrm{Ohr}$ für das Verstehen der Sprache gentigt, so sind beide Acustici mindestens mit diesem Theil des linken Schläfelappens in Verbindung.

Nun kommt, wie aus der Beobachtung pathologischer Fälle wie des Lichtheim'schen und des unserigen, die hier das Experiment ersetzen müssen, hervorgeht, bei Gebirnkrankbeiten als alleiniges Ausfallssymptom zwar Verlust des Hörvermögens für die Sprache und die Musik vor, niemals aber wurde als einziges Ausfallssymptom Verlust des übrigen Hörvermögens bei erhaltenem Hörvermögen für die Sprache und die Musik beobachtet; war ersteres central gestört, dann war es auch gleichzeitig das letztere ${ }^{3}$ ). Daraus kann nur geschlossen werden, dass die sensible Leitungsbahn für das im linken Schläfe-

1) Fortscbritte der Medicin. 1883. Nr. 6.

2) Sachs, Das Gehirn des Förster'schen "Rindenblinden“. (Arbeiten aus der psychiatrischen Klinik zu Breslau. Heft II. S. 89.)

3) Vgl. unten bei der Casuistik Fall 5 ( $\mathrm{Pick}$ ); ferner den obigen Fall von Friedländer und Wernicke. 
lappen gelegene akustische Sprachcentrum das allgemeine Hörcentrum passirt.

Demnach ergiebt sich für diese Vorgänge das nachstehende Schema (Fig. 3). $H_{1}$ und $H_{2}$ entsprechen den Rindenfeldern für das Hören in beiden Schläfelappen. $\mathrm{Zu}$ ihnen ziehen halb gekreuzt die beiden Hörnerven vom linken $\left(A_{1}\right)$ und vom rechten $\left(A_{2}\right)$ Gehörorgan. $W$ ist das Rindenfeld für die Sprache im hinteren Theil der ersten und zweiten linken Schläfewindung. $H_{1} W$ und $H_{2} W$ sind die zu ihm aus $H_{1}$ und $H_{2}$ führenden Bahnen. $H_{2} W$ muss eine durch den Balken (Tapetumbahn?) führende Bahn sein; $\mathrm{H}_{1} \mathrm{H}_{2}$ stellt die beide Hörsphären miteinander verkntipfende Associationsbahn (vordere Commissur?) vor. Wentspricht dem Punkte $A$ in dem Schema Lichthei m's, die beiden Bahnen $H_{1} W$ und $H_{2} W$ zusammen dessen Bahn $a A$. Das Krankheitsbild der subcorticalen sensorischen Aphasie kann bei einheitlichem Herd nur entstehen durch Unterbrechung: beider Bahnen an der Stelle, wo sie kurz vor ibrer Einmündung in $W$ dicht bei einander liegen, oder

Fig. 3.

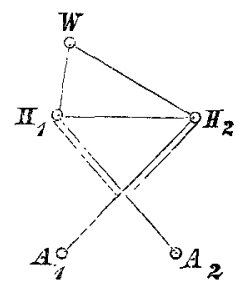
durch doppelseitige Herde, die jede Bahn für sich unterbrechen. Beide Momente scheinen mir die Seltenheit des reinen Krankheitsbildes genügend zu erklären. Da es sich dabei um die Unterbrechung von Leitungsbahnen handelt, so erklärt sich auch die Unveränderlichkeit des Krankheitsbildes und die Unmöglichkeit einer Ausgleichung. Eine solche könnte nur stattfinden, wenn eine indirecte Lähmung dieser Bahn vorliegt. Ich glaube dafür unten ein Beispiel aus der Casuistik beibringen zu können. Störungen des Centrum $W$ werden dem gegeniber bekanntlich leicht ausgeglichen; denn alle Autoren sind darin einig, dass das Symptom der Worttaubheit, dass ja meist auf eine solche Läsion zurückzuführen ist, sich gewöhnlich schnell wieder verliert. Auch schon aus diesem Grunde ist man, wie ich meine, gezwungen, für unseren obigen Fall eine Leitungsunterbrechung anzunehmen; denn da die Sprachtaubheit so gut wie ganz obne Allgemeinerscheinungen einsetzte, so kann es sich sicher aur um einen kleinen Krankheitsherd gehandelt haben. Verlegt man diesen in das Centrum $W$, so bleibt gegenuber anderen Fällen das Ausbleiben jeder Spur von Rückbildung in der Sprachtaubheit ganz räthselhaft. Mögen auch sonst gegen obige Darstellung Einwendungen erhoben werden, in diesem Punkte scheint mir eine abweichende Erklärung kaum möglich zu sein. Alsdann aber kommt man nothwendig zur Annahme einer subcorticalen sensorischen Aphasie. Der Ausdruck „subcortical“ 
hat dabei nach obigem Schema allerdings nur eine bildliche Bedeutung für die Bahn in Bezug auf das Centrum $W$, in Wirklichkeit ist es eine intercentrale, d. b. eine Associationsbahn. Lichtheim's Darstellung finde ich in Bezug auf diesen Punkt dunkel. Er will die Affection periphere Leitungssprachtaubheit nennen und meint, dass sie nur dann entstehen könne, wenn die Einstrahlungen beider Acustici in den linken Schläfelappen durchbrochen seien. Woher aber diese Einstrahlungen kommen, ob von einem anderen Centrum oder ob direct vom $\mathrm{Ohr}$, ist mir aus seiner Darstellung nicht klar geworden. Uebrigens ist auch eine andere subcorticale Bahn Wernicke's, nämlich die für die subcorticale Alexie, meiner Ansicht nach eine intercentrale. Wie ich mich an einem ganz reinen Fall von Wortblindbeit ${ }^{1}$ ) iberzeugt habe, sind beide Krankheitsbilder theoretisch einander völlig analog $\mathrm{zu}$ erklären.

Von dem Punkte $W$ geht die Lichtheim'sche Bahn $A M$ aus, deren Störung wir, wie oben ausfluhrlich gezeigt wurde, zur Erklärung einiger Symptome bei unserem Kranken heranziehen mussten. Da, wie schon oben erwähnt, der Annahme nichts entgegensteht, dass die in $W$ einmiundenden Bahnen einander wenigstens dicht vor der Einmündung benachbart sind, so würden sie durch einen einbeitlichen, nicht sehr umfangreichen Blutungsherd, wie wir ihn nach den klinischen Erscheinungen voraussetzen mussten, gemeinsam betroffen werden können. Für unseren Fall ist somit Theorie und Beobachtung auch hier in befriedigender Uebereinstimmung. Da von dem Punkt $W$ auch die Lichtheim'sche Bahn $A B$ ausgeht, so ist der Theorie nach auch das Zusammenvorkommen von subcorticaler und transcorticaler sensorischer Aphasie möglich. In der That ist das daraus resultirende Krankheitsbild schon beobachtet worden. ${ }^{2}$ )

Nach den vorausgegangenen Anschauungen dürften über das Functioniren des von uns oben gegebenen Schemas nur noch wenige Bemerkungen erforderlich sein. In der Schnecke erregt nach der gegenwärtig allgemeinen Annahme ein bestimmter Ton jedesmal allein oder vorwiegend einen einzigen bestimmten auf ihn abgestimmten nervösen Endapparat, dessen Erregung durch eine bestimmte von ihm ausgehende Nervenfaser auf eine oder vermuthlich durch Mitwirkung von Collateralen auf mehrere Ganglienzellen in der Rinde des Schläfelappens übertragen wird. Da bei allem, was wir hören, niemals ein-

1) Ueber diesen Fall wurde von mir auf der Naturforscherversammlung in Lübeck kurz berichtet. Vielleicht finde ich einmal Gelegenheit, Ausführliches über ihn mitzutheilen.

2) Vgl. u. Fall 3 (Adler). 
fache Töne, sondern stets eine Summe von solchen mitwirkt, so findet bei jedem Höract stets eine Erregung einer ganzen Zahl von Ganglienzellen statt, die man sich in bisher nicht genau bekannter Weise mit einander verbunden vorstellen muss, so dass eine einheitliche Erregung resultirt, deren psychisches Correlat eine akustische Wahrnehmungsvorstellung ist. Die Erregung hinterlässt nach der ublichen Annahme in den einzelnen Ganglienzellen eine physische Disposition nicht nur für Erregungen derselben Art, sondern auch dafür, sich zu gleichen Verbindungscomplexen zu vereinigen. Beides ist als die materielle Grundlage des Gedächtnisses anzuseben; psychisch entspricht es ibr, dass fernere Erregungen dieses Complexes den Charakter von Erinnerungsvorstellungen haben, oder wie $\mathbf{R} \mathbf{i b}_{\mathbf{b}} \mathbf{t}^{1}$ ) dieses ausdrückt, „das organische Gedächtniss setzt nicht nur eine Modification der Nervenelemente voraus, sondern auch die Bildung von Associationen unter ihnen für jedes besondere Ereigniss, die Herstellung bestimmter dynamischer Associationen, welche durch die Wiederholung ebenso beständig werden, wie die gegebenen anatomischen Verbindungen".

Wenden wir dies auf das Hörcentrum an, so gehen die erforderlichen centralen physiologischen Erregungen und Verbindungen für die akustischen Wahrnehmungs- und Erinnerungsvorstellungen in den Punkten $H_{1}$ und $H_{2}$ vor sich. Nun macht das Krankheitsbild der subcorticalen sensorischen Aphasie es einerseits wahrscheinlich, dass auch die Vokale, Consonanten und musikalischen Töne hier gehört und erkannt werden, andererseits beweist es aber, dass für deren Verbindungen zu Wörtern und Melodien anderswo localisirte Zellcomplexe vorhanden sein müssen, die dem Punkte $W$ unseres Schemas entsprechen. Wäbrend in den zu $H_{1}$ und $H_{2}$ führenden Nervenfasern einfachen Empfindungen entsprechende Erregungen stattfinden, ist dies in den zu $W$ fuhrenden Nervenfasern nicht mehr der Fall; man muss vielmehr annehmen, dass durch ihre,Vermittlung den Ganglienapparaten des Centrums $W$ die in $H_{1}$ und $H_{2}$ geschehene Erregung von ganzen Zellgruppen signalisirt wird, die den Sprachelementen und musikalischen Tönen entsprechen. Die neneren mikroskopischen Befunde iber die Structur der nervösen Centralorgane lassen derartige Annahmen auch anatomisch als zulässig und leicht verständlich erscheinen. In dem Centrum $W$ geschieht also infolge von Association dieser successive signalisirten Erregungscomplexe deren Verknüpfung und Verschmelzung zu einem neven einheitlichen Erregungsvorgang,

1) 1. c. S. 12 . 
dem die Wortvorstellung entspricht. Ganz ähnlich erklärt Ribot das Entstehen von secundären automatischen Bewegungen, z. B. das Tanzen daraus, "dass bestimmte Modificationen der bei der Ortsbewegung gebrauchten Gelenke und Muskeln schon in gewissen Nervenelementen registrirt sind: jene Bewegungen finden mit einem Wort ein bereits organisirtes Gedächtniss vor, von dem sie mehrere Elemente zur Bildung einer neuen Combination und eines anderen Gedächtnisses zu ihren Gunsten verwenden".

Das Krankheitsbild, welches uns hier beschäftigt, ist deshalb, wie mir scheint, ein interessantes und theoretisch wichtiges, weil es, wie die vorstehende Analyse zeigt, einen Einblick in das Zustandekommen complicirter psycho-physischer Vorgänge gewährt. Nur aus einer möglichst gründlichen Beobachtung solcher pathologischer Fälle beim Menschen, deren Eintritt ja mehr oder weniger immer von einem glïcklichen Zufall abbängt, können wir derartige Einblicke gewinnen. Sie müssen die Beweisstücke für manche psycho-physiologischen Hypothesen bilden. Wir glauben hier den Beweis für eine solche schon von Kussmaul') ausgesprochene Vermuthung geliefert zu haben: das Wort als sensorischer Act könne ein Complex von Empfindungseinheiten sein, die von unten nach oben in wachsender Gliederung sich zusammenordnen.

\section{Casuistik.}

Die Casuistik der subcorticalen sensorischen Aphasie ist noch eine sehr spärliche. Seit dem Jahre 1885, wo Lichtheim seinen oben erwähnten Fall veröffentlichte, sind nur ganz wenige Fälle mit dieser Diagnose beschrieben worden. Ja, einer von diesen Fällen ist sogar, wie wir später sehen werden, kürzlich wieder zurückgezogen und anders gedeutet worden. Es scheinen mir jedoch schon aus der Zeit vor Lichtheim einige Beobachtungen vorzuliegen, in denen man zweifellos das Bild der subcorticalen sensorischen Aphasie erkennen kann. Eine weit grössere Zahl dagegen bleibt zweifelhaft, weil die Krankengeschichten, wie schon Lichtheim richtig bemerkt, viel zu wenig genau sind, als dass man ein Urtheil gewinnen könnte. Um subcorticale sensorische Aphasie scheint es sich mit mebr oder weniger grosser Wahrscheinlichkeit in den nachfolgenden Fällen gehandelt zu haben, die ich glaube anführen zu sollen, da sie als weitere Belege für einige meiner obigen Ausführungen dienen können und noch zu einigen speciellen Bemerkungen Gelegenheit geben.

1) 1. c. S. 102 . 
1. Beobachtung von Kahler und Pick (Vierteljahrsschrift für prakt. Heilkunde. Bd. CXLI. S. 24).

Den Patienten kannte sein erwachsener Sohn nie anders als angeblich taub und unverständlich sprechend. Da die Mutter während seiner Kindheit verstorben war, konnte der Sohn Angaben über die Entstehung des Leidens nicht machen. Patient reagirt nur auf starkes Anrufen. Das gesprochene Wort versteht er nicht, die Untersuchung zeigt jedoch, dass die Gehörsempfindung als solche erhalten ist. Gesten und selbst die Mund- und Lippenbewegungen fasst er richtig auf. Ohne Zuhülfenahme des Gesichts ist das Verständniss des Gesprochenen immer und vollständig unmöglich. Melodien allbekannter, ihm vorgesungener Lieder kennt er nicht; doch ist er sich bewusst, dass gesungen wird. Seine Sprache besteht aus zusammenhängenden Worten und Silben, mit denen er seine Gedanken, die er mimisch recht gut kund zu geben weiss, auszudrücken trachtet. Mitunter gelingt es ihm, einen vorgehaltenen Gegenstand zu benennen. Die Sprachversuche werden öfter's von interjectionellen Sätzen wie: „Ach, Herr Jesus, ich weiss nicht was" nnterbrochen. Beim Lesen bringt er im Ganzen lauter. Silbenzusammenstellungen, die einige Aehnlichkeit mit dem Vorgehaltenen haben, dagegen namentlich in den Consonanten verdreht erscheinen. Die Schrift ist gut und sicher, seinen Namen schreibt er spontan auf und ist dann auch im Stande ihn zu nennen, sonst schreibt er nur unzusammenhängende Silben. Vorgeschriebenes schreibt er nach. Löscht man das Wort aus, so gelingt das Nachschreiben nicht vollständig, er findet nur noch den Anfang des Wortes wieder. Aufgeschriebene Fragen bleiben unverstanden. Eine Untersuchung auf der otiatrischen Klinik ergab nur eine mässige Einziehung beider 'Trommelfelle, die nicht die Grenzen der Norm iberscbritt. Den recbten Arm trug er in einer posthemiplegischen Haltung. - Kahler und Pick machen besonders aufmerksam auf die Unveränderlichkeit des Krankheitsbildes, ferner darauf, dass der Patient, dessen Gehör sowohl nach den Ergebnissen der functionellen Prüfung als nach der ohrenärztlichen Untersuchung intact war, den Sinn des Gehörten absolut nicht versteht und ihm bekannte Lieder nicht percipirt, obwohl er Töne hört, ein, wie sie sagen, bisher in dieser Form noch nicht beobachteter Defect des musikalischen Verständnisses. Da eine Ausgleichung nicht erfolgte, was bei einer wirklichen unheilbaren Zerstörung der betreffenden Hirnabschnitte durch vicariirendes Eintreten der anderen Hemisphäre ihrer Ansicht nach hätte geschehen miissen, so nehmen sie eine symmetrische Affection an. Der rechtsseitige Herd ist jedoch reine Hypothese, während auf den linksseitigen auch der noch vorhandene Rest einer früheren rechtsseitigen Hemiplegie hindeutet. Nach unserer Ansicht, die nach dem Vorausgegangenen kaum einer ausführlichen Begründung bedarf, handelt es sich in dem Fall um eine Combination von transcorticaler und subcorticaler sensorischer Aphasie. Besonders die letzte erkennt man leicht in der Krankengeschichte. Eine doppelseitige Läsion beider Schläfelappen hätte noch andere Hörstörungen als nur Sprachtaubheit hervorrufen müssen (vgl. Fall 5).

2. Beobachtung von Schmidt (Allgemeine Zeitschrift für Psychiatrie. Bd. XXVII. S. 304).

Eine 25 jährige Frau wurde 8 Tage nach der Entbindung plötzlich 
bewusstlos. Sie kam bald wieder zur Besinnung, konnte aber Worte schwer hervorbringen, und verstand nur mit Mühe nach mehrmaliger Wiederholung einzelne Worte. Am folgenden Tage verstand sie kein Wort, wenn man auch noch so laut sprach. Beim Sprechen musste sie sich anstrengen, um ein Wort zu finden und es auszusprechen, zuweilen verstümmelt sie es oder gebraucht ein ganz anderes Wort, die Silben waren oft nicht richtig, Buchstaben fielen aus u. s. w. Das Sehen war gut, das Bewusstsein hatte nicht gelitten. Zur Verständigung mit ihr diente die Schrift. Sie betrachtete ein Wort nach dem anderen, suchte sie einzeln und dann auch im Zusammenhang auszusprechen und dann zu antworten. Man hielt sie für taub, weil sie kein Wort verstand, uberzeugte sich aber bald, dass sie das Klopfen an der Thtir u. s. w. so scharf hörte wie ein Gesunder $\mathfrak{u}$. dgl. m. Alle einfachen, auch sich wiederholenden Klänge hörte sie. Sie hörte einzelne Vokale und sprach sie nach. Sprach man in gewöhnlicher Weise ein einsilbiges Wort, so verstand sie es nicht, trennte man aber die einzelnen Buchstaben scharf von einander, so dass sie in der Aussprache deutlich hervortreten, so sprach sie es nach; bei mehrsilbigen Wörtern musste man zuerst eine Silbe deutlich aussprechen, dann die andere ebenso, dann erst beide zusammen, wenn sie das Wort verstehen sollte. Erst nach einem halben Jahr verstand sie bei deutlicher langsamer Aussprache einen ganzen, auch nur kurzen Satz ohne Wiederholung. Auch die Sprachfertigkeit nahm zu, doch blieb die Sprache immer etwas Miuhsames. Sie äusserte später, sie habe beim Sprechen wohl gehört, sie habe aber nichts als ein verworrenes Geräusch vernommen. Schmidt macht zu seinem Fall folgende Bemerkungen: Da Patientin einzelne, gleichmässige Klänge und Laute ganz gut hörte, musste der Grund, dass sie ausgesprochene Wörter nicht verstand, darin liegen, dass sie die einzelnen Buchstabenlaute nicht schnell genug nach einander auffassen und zu einem Worte vereinigen konnte. Es musste also dasjenige Organ im Gehirn gelitten haben, welches die Function hat, die Laute zu combiniren und das Klangbild herzustellen. Auf Grund dieser von ihm citirten Beobachtung kommt auch Kussmaul zu dem Schluss, dass die Wahrnehmung von Klängen und Geräuschen, die für sich als Vokale und Consonanten begriffen werden, und ihre Fiigung zum akustischen Wortbild, das als Symbol dieser oder jener Vorstellung erfasst wird, verschiedene Functionen seien, die an verschiedene Centraltheile gebunden wären. Merkwürdiger Weise citirt Lichtheim den Fall als reine transcorticale sensorische Aphasie, während doch das ganze Verbalten der Kranken beim Hören, ferner der Umstand, dass sie für taub gehalten wurde, seinen darauf beztiglichen Anforderungen widerspricht und die Kranke mit seinem eigenen Fall von subcorticaler sensorischer Aphasie in Parallele bringt. Leider fehlen Angaben iiber das musikalische Verständniss. Mir scheint in dem Fall das Wesentliche eine subcorticale sensorische Aphasie gewesen zu sein, combinirt mit transcorticaler oder mit Leitungsaphasie. Jedoch war sie hier indirectes symptom und infolge dessen nicht complet.

3. Beobachtung von Adler (Neurolog. Centralbl. 1891. S. 294). 47 jähriger Mann. 1888 plötzliches Auftreten der Sprachtaubheit unter Schwindel ohne Bewusstseinspause. Sprache fliessend, nur zuweilen findet 
er ein Wort nicht oder verwechselt es. Verstand nicht mehr, was er las; Schreibvermögen hochgradig gestört. Die Sprachtaubheit verschwand im Laufe eines Jahres, die Störungen der willkürlichen Sprache, der Schrift und des Schriftverständnisses besserten sich nur wenig. 1890 furchtbare Kopfschmerzen, 'Taubheit für eine Woche, dann wenige Tage Besserung, Auftreten einer linksseitigen Lähmung, einige Wochen hindurch Delirien. November 1890 folgender Befund: Völlige Sprachtaubheit, versteht kein einziges Wort, wenn man es auch noch so laut und deutlich ausspricht, ,ja, hören thu' ich's, aber ich versteh's nicht". Schallerscheinungen gegenüber besteht eine sehr geringe Aufmerksamkeit gerade so, als ob er taub wäre; er wendet sich nicht um, wenn hinter seinem Rücken laut gesprochen oder irgend ein Geräusch erzeugt wird. Hörprüfung: Uhr rechts $1 \mathrm{Cm}$. vom Ohr, links im Contact; Flisterstimme beiderseits $6 \mathrm{M}$., laute Sprache $15 \mathrm{M}$. Stimmgabel vom Scheitel nach links, Rinne links negativ, rechts positiv. Kein defect für hohe Töne. Also chronischer Mittelohrkatarrh, jedoch erklärt die dadurch erzeugte Verminderung der Hörschärfe nicht die Sprachtaubheit. Andere Schallerscheinungen als die Sprache fasst er richtig auf, unterscheidet musikalische Instrumente ihrem Klange nach, Melodien erkennt er nicht, singt sie auch nicht mit. Nachsprechen nicht möglich. Sprache: fliessend und richtig, bei vorgezeigten Gegenständen findet er zuweilen schwer den Namen, obwohl er sie erkennt, öfters Paraphasie dabei. Lesen fliessend, zwar langsam, aber meist fehlerfrei mit monotoner Stimme ohne jede sinngemässe Betonung der einzelnen Worte, weil das Verständniss fast fehlt. Ziffern liest er richtig. Abschreiben von Druckschrift mangelhaft, von Currentschrift gut, schriftliche Bezeichnung von Objecten hochgradig gestört, da er viele Buchstaben nicht finden kann. Die Schriftstörung war nach A dler nur zum kleinsten Theil durch fehlerhaftes Buchstabiren bedingt, beruhte vielmehr auf der mangelhaften Erregung der optischen Buchstabenbilder. Ophthalmoskopisch bestand beiderseitige, sehr starke concentrische Gesichtsfeldeinengung, trotzdem war das Orientirungsvermögen intact (Anaesthesia retinae). Keine motorischen Störungen, nur links Händedruck schwächer. - Adler hält den Fall für eine Combination von subcorticaler und transcorticaler sensorischer Aphasie; die Schriftstörung sammt der Anaesthesia retinae bezieht er auf eine Herabsetzung der Erregbarkeit der optischen Rindenfelder. Er nimmt an, dass der erste Schlaganfall die transcorticale sensorische Aphasie bewirkt habe, die sich bis auf die Paraphasie, die Lese- und Schreibstörungen wie in anderen Fällen zuriickbildete; erst der zweite Anfall machte die subcorticale sensorische Aphasie. Von der begleitenden linksseitigen Hemiplegie, meint Adler, sei es höchst wahrscheinlich, dass sie nicht gleichzeitig mit der Sprachtaubheit entstanden sei, ,ich sehe nämlich keine Möglichkeit, eine derartige Combination bei dem rechtshändigen Manne za erklären". Unser obiges Schema hilft leicht über diese Verlegenheit weg. Der erste linksseitige Herd bewirkte durch Störung der transcorticalen Lichtheimschen Bahn $A B$ die Paraphasie und die Störungen der Schriftsprache, ferner die Worttaubheit durch directe Läsion unserer Bahn $H_{1} W$ und indirecte Läsion der Bahn $H_{2} W$, indem wir ihn in die Nähe der Einmiłndungsstelle jener drei Bahnen verlegen. Indem die indirect gelähmte 
Strecke $\mathrm{H}_{2} \mathrm{~W}$ sich erholte, verschwand die Worttaubheit wieder. Nach 2 Jahren wurde sie von einem rechtsseitigen Herd zerstört, der zugleich linksseitige Hemiplegie machte, und nun entstand von Neuem Worttaubheit, da die linksseitige Bahn $H_{1} W$ bereits von dem früheren Herd zerstört war. Die subcorticale sensorische Aphasie tritt jedenfalls deutlich in dem Krankheitsbild hervor.

4. Beobachtung von Cramer (Archiv für Psychiatrie. Bd. XXII. S. 141).

Patient galt seit mehreren Jahren als verdrehter Herr. Die Hörfähigkeit war herabgesetzt. Flüstersprache wurde rechts auf $80 \mathrm{Cm}$., links nur auf $40 \mathrm{Cm}$. Entfernung vernommen. Beide Trommelfelle leicht getrübt und eingezogen. Es bestand Paraphasie, Verarmung des Wortschatzes, viele Gegenstände konnte er nicht bezeichnen. Ferner zeigte sich Mangel an Wortverständniss: „,Was? Was heisst das? Was soll das heissen? Das verstehe ich nicht. Diese Aussprache verstehe ich nicht" ". Derartige Wendungen gebrauchte er oft, wenn man langsam, laut und deutlich mit ihm sprach. Besonders für Hauptwörter war der Mangel an Verständniss auffallend. Vorgesprochene Hauptworte (Bezeichungen von Gegenständen, die er nicht zu nennen vermag) kann er nicht oder nur fehlerhaft nachsprechen. Einzelne Buchstaben las er richtig, ganze Wörter aber oft verkehrt. Ebenso bestanden Schriftstörungen, lässt Wörter und Buchstaben aus, gebraucht falsche Worte, im Wort falsche Buchstaben u. s. w. Die Buchstabenbildung selbst war richtig. Dictatschreiben, das noch durch die Störungen des Wortverständnisses erschwert wurde, erfolgte mit Paragraphie. Auch Symptome von Seelenblindheit waren vorhanden. Während der $31 / 2$ jälırigen Beobachtungszeit bildeten die Worttaubheit und die Paraphasie die constantesten und auffälligsten Erscheinungen. Die Section ergab eine Herderkrankung im linken Schläfelappen, vorzugsweise im Mark und der Rinde des hinteren $2 / 3$ der ersten und der Randzone der zweiten Schläfenwindung, auch die Insel und das tiefe Mark des unteren Scheitelläppchens war betheiligt. - Es soll nicht bestritten werden, dass, wie Cramer annimmt, an dem Krankheitsbild subcorticale sensorische Aphasie betheiligt war. Die Beschreibung giebt jedoch von der Art und dem Grade der Worttaubheit kein ganz klares Bild; vor Allem erkennt man aus den spärlichen Angaben nicht genügend, wie weit das Ohrleiden des Patienten daran betheiligt war. Der auch im Uebrigen sehr complicirte Fall erlaubt also keine Schlüsse und ist hier nur deshalb angeführt, weil sein Verfasser ihn direct als hierher gehörig bezeichnet. Ueber das Musikverständniss fehlen Angaben.

5. Beobachtung von Pick (Archiv für Psychiatrie. Bd. XXIII. S. 902).

Geisteskranker Patient, hatte wiederholt Schlaganfälle gehabt, unter anderen auch mit linksseitiger Hemiplegie, der linksseitige untere Facialisast war noch gelähmt. Das Sprachverständniss fehlt ganz, Fragen beantwortete er falsch, insofern er correcte, aber nicht zur Sache gehörige Aeusserungen macht. Er machte den Eindruck eines Stocktauben, auf Geräusche in seiner Umgebung achtet er gar nicht, er percipirt indessen leiseres Rufen, Sprechen u. s. w. Das Verständniss gehörter Me- 
lodien schien gleichfalls gestört, er konnte aber etwas Flöte spielen. Sprache correct, vorgezeigte Gegenstände bezeichnet er richtig. Die Schrift war langsam, aber correct, ebenso das fliessend von Statten gehende Lesen, für welches das Verständniss erhalten war. Nachsprechen, Dictatschreiben fehlt vollkommen. Der Zustand des Patienten änderte sich während der Beobachtungzeit nicht. Section: Rechts Gyrus temp. I. und ziemlich grosse Theile des Gyr. temp. II, ferner die ganze Insula Reilii und kleine umschriebene Stellen im unteren Ende der vorderen Centralwindung und in der untersten Stirnwindung erweicht (Rinde und Mark). Links die hintere Hälfte des Gyr. sphenoidalis I und des Gyr. supramarginalis erweicht. - Pick hebt mit Recht die Aehnlichkeit mit dem Lichtheim'schen Fall hervor, und fasst den Fall auf als reine Worttaubheit, neben der ein mittlerer Grad von Taubheit vorhanden war. Er glaubt, eine subcorticale Form der Sprachtaubheit annehmen zu müssen; die Schwerhörigkeit, die leider nicht genau untersucht worden ist, sei wohl auf die centrale Affectien zu beziehen. Angesichts dieses Falles dränge sich die bisher wenig discutirte Frage nach den Beziehungen der Worttaubheit zur Taubheit auf; auch scheine der Erwägung bedürftig, in wiefern eine von Siemerling gegebene Deutung einzelner Fälle von Seelenblindheit auch für die Worttaubheit Anwendung finden könne. Bei dem Vergleich mit dem Lichtheim'schen Falle darf jedoch nicht übersehen werden, dass der Patient von Pick geisteskrank war, seine Intelligenz hatte gelitten, sein Verhalten war kindisch, seine Aufmerksamkeit war nur schwer für einige Zeit zu fixiren; die meiste Zeit verbrachte er mit Beten, in welcher Beschäftigung ihn keinerlei Lärm in der Umgebung: stören konnte. War nicht zum Theil der Anschein der Taubheit auf die Geisteskrankheit zu beziehen? Auch fehlt jede Untersuchung des Ohres. Der Fall ist also keineswegs ganz klar und sicher, auch verhielt er sich in einem wichtigen Punkte ganz anders als der Lichtheim'sche Patient; er beantwortete nämlich Fragen falsch, insofern er correcte, aber nicht zur Sache gehörige Aeusserungen machte. Demnach musste er doch die Empfindung gehabt haben, dass er sprachlich angeredet worden war. Der Lich the im'sche und unser eigener Patient antworteten tiberhaupt nicht. Ich habe daher von dem Fall eine etwas andere Auffassung.

Ich stimme Pick bei, dass in seinem Falle die Schwerhörigkeit von der durch die Section nachgewiesenen doppelseitigen Gehirnaffection abhängig war. Nach der von mir gegebenen Darstellung muss aber in einem solchen Falle neben den ibrigen akustischen Wahrnehmungs- und Erinnerungsvorstellungen stets auch die Wahrnehmung der Buchstaben gestört sein; denn da die an der Perception betheiligten Ganglienzellen in $H_{1}$ und $H_{2}$ theils zerstört, theils in ihrer Function geschädigt sind, so wird auch der zur Entstehung des Buchstabenklangbildes führende Associationsvorgang mehr oder weniger gestört sein, so dass an Stelle der normalen Sprachlaute mehr oder weniger fremdartig klingende Elemente gebildet und nach $W$ signalisirt werden. Daraus aber muss sich eine Störung des Sprachverständnisses ergeben, deren Grad von dem Grade jener anderen Störung ablängig ist. So erklärt sich ganz befriedigend der Unterschied in dem Krankheitsbild, den der Kranke Pick's beim Anreden gegenjiber dem Lichtheim'schen und unseren eigenen Kranken zeigte; eigent- 
liche subcorticale sensorische Aphasie lag somit nicht vor, da man diesen Namen doch wohl auf die Störung der Bahn $H_{1} W$ und $H_{2} W$ beschränken muss. Für die Störung des Sprachverständnisses bei dem Pick'schen Kranken wäre ein Name noch erst zu bilden (vielleicht centrale sensorischakustische Aphasie?).

6. Beobachtung von Bernard (De l'aphasie. Paris 1889. Observ. XII. p. 146).

49 jährige Patientin kommt in das Spital wegen einer Obliteration der Aorta abdom. Seit 6 Jahren rechtsseitige Hemiplegie mit Contracturen, 2 Jahre hindurch konnte sie nur "oui" und "non" sagen. Dann stellte sich das Sprechvermögen allmählich völlig correct wieder her. Sie schrieb etwas mit der linken Hand, lesen hatte sie immer können. Sie verstand alle Fragen und ihre Antworten zeigten keine Incorrectheit. Im Anfang von ihrer Hemiplegie hatte sie jedoch nur zuweilen die an sie gerichteten Worte verstanden, man hatte sie mehrmals wiederholen milissen, und sie wusste genau die Personen zu fixiren, die mit ihr sprachen. Ihre Umgebung hielt sie für taub. Die Worte waren ftir sie nur ein undeutliches Geräusch, ähnlich dem von einem Haufen redender Menschen. Sie hörte indessen allerlei Geräusche, das Anklopfen an die Thtir u. s. w. Wenn Militärmusik spielte, hörte sie wohl, dass sich ein neues Geräusch denen zufügte, welche sie gerade vernahm, aber es war nur ein Geräusch gänzlich der Melodie entbehrend. Section: In der linken Hemisphäre ein ausgebreiteter Erweichungsherd, der den vorderen Theil und den Fuss der dritten Stirnwindung, die ganze Insel, die ganze erste Temporalwindung und einen kleinen Theil des unteren Parietallappens zerstört hatte. Auch der ganze Kopf vom Nucleus caudatus und das vordere Drittel des Thalamus opticus sind zerstört, desgleichen die Vormauer und der Linsenkern mit Ausnahme seines hinteren Theiles, der an den Carrefour sensitif grenzt, welcher intact ist, ebenso wie die anstossende Partie des Thalamus und der Schwanz des Corpus striatum. Der ganze ubrige Theil der inneren Kapsel und die vorderen Theile der centralen Ganglien sind in eine gelbliche Masse verwandelt. Das rechte Gehirn ist normal. -Die subcorticale sensorische Aphasie tritt in dem Falle ganz deutlich hervor, die wenigen Worte, die sie zuweilen verstand, hatte sie offenbar vom Munde abgelesen. Nach dem Sectionsbefund muss man annehmen, dass der Ausgleich sowohl der sensorischen als der motorischen Aphasie durch allmähliches Eintreten der rechten Hemisphäre erfolgt war, daher auch die lange Dauer von 2 Jahren, bis der Ausgleich ein vollständiger war.

7. Beobachtung von Edgren (Zeitschrift für Nervenheilkunde. Bd. VI. S. 41. Fall 52).

34 jähriger Patient, mehrere Wochen nach einem Kopftrauma mit verschiedenen cerebralen Symptomen erkrankt, kann Musik nicht auffassen; er höre sie wohl, aber nicht wie gewöhnlich, sondern wie ein unbestimmtes Geräusch, so dass er gar keine Melodie herausfinden könne. Als seine Frau zu ihm sprach, verstand er sie nicht. Nachdem die Sprache 2 Tage gefehlt hatte, sprach er verwirrt und unzusammenhängend. Constantes Symptom ist Worttaubheit. Ab und zu versteht er einzelne Worte. Nachsprechen fehlt, er sagt, dass er höre, wenn man zu ihm spreche, es aber 
nicht verstehe. Eine schriftliche Mittheilung versteht er, wenn sie nicht zu verwickelt ist. Die Sprache meist fliessend, zuweilen paraphasisch. Laut lesen leicht und correct, nach einer Weile aber paraphasisch. Schreiben anfangs richtig und mit guter Handschrift, bald aber werden Buchstaben zugesetzt, weggelassen u.s. w., oft ist die Schrift völlig verworren, oft aber auch völlig fehlerfrei und tadellos. Das Gehör ist ein wenig herabgesetzt, auf beiden Seiten gleich. Nach einer Woche versteht er ziemlich gut, wenn man mit ihm über etwas spricht, 'womit seine Gedanken gerade beschäftigt sind. Auf andere Fragen reagirt er nicht, sondern fährt über den früheren Gedanken za sprechen fort, unbekümmert, ob Jemand auf ihn hört. Wenn man ihn alsdann schittelt oder sehr markirend und langsam mit ihm spricht, so verstebt er bisweilen die nene Frage. Später versteht er fast immer, wenn man seine Aufmerksamkeit erregt und langsam und deutlich spricht, er kann jetzt auch nachsprechen und auf Dictat schreiben. Schliesslich verschwand die Worttaubheit, nur geringe Paraphasie blieb nach. Ebenso blieb er tontaub. Sein früher gates musikalisches Gehör war völlig verschwunden, so dass ein Orchester nur wie ein Geräusch aufgefasst wurde und er nicht zwischen Walzer, Polka oder Marsch unterscheiden konnte. Am Gesangverein konnte er nicht mehr theilnehmen, weil er die Musik nicht mehr auffasste. Als er vor seinen Kindern singen wollte, wie er früher häufig zu thun pflegte, verlor er bald die Melodie, weil er seinen eigenen Gesang nicht auffasste. Noten hatte er nicht gelernt, auch spielte er kein Instrument. Nach 2 Jahren Tod an Purpura. Section: Frische Pachymeningitis interna haemorrh., ältere Erweichungsherde in der Oberfläche der Gehirnhemisphären: Links in dem vorderen Theil der Fossa Sylvii eine Einsenkung, die die vorderen zwei Drittel der ersten Temporalwindung and die vordere Hälfte des Gyrus temporalis medius umfasst. An dem Uebergange zwischen dem hinteren und dem mittleren Drittel des Gyr. tempor. sup. war die oberflächliche Schicht sklerotisch und mit der Pia verwachsen. Unter diesem sklerotischen Theil und $1 \mathrm{Cm}$. weiter nach hinten geht die Erweichung auch in das Mark der ersten Temporalwindung. Die umliegenden Theile der Gehirnrinde waren gesund. In der rechten Hemisphäre um den R. horizontalis fossae Sylvii ein ähnlicher Defect. In der Rinde sind zerstört die obere und äussere Oberfläche der hinteren Hälfte des G. temp. sup. und entsprechend der untere Rand des G. supramarginalis. Die Zerstörung ging im Boden der Fossa Sylvii in die weisse Substanz über und war nach vorn und nach hinten ausgedehnter als in der Rinde.

Die subcorticale sensorische Aphasie war hier nur ein voriibergehendes, indirectes Symptom; obschon nicht gerade sehr deutlich beschrieben, erkennt man sie doch aus einigen Sätzen der Krankengeschichte mit Deutlichkeit (man musste ihn erst schtitteln, damit er aufpasste und dann langsam und deutlich sprechen, worauf er bisweilen verstand). Besonders interessant ist aber, dass die auch sonst häufig damit verbundene Tontaubheit dauernd bestehen blieb. Wir kommen später darauf zurtick. Trotzdem nicht unerhebliche Theile von beiden Schläfelappen zerstört waren, muss der Rest zum Verständniss der Sprache genügt haben, da diese Störungen sich bald ausglichen. Bemerkenswerth aber ist, dass gerade das hintere Drittel der ersten Temporal- and die hintere Hälfte 
der zweiten Temporalwindung links intact waren. Ferner war rechts derjenige Theil erhalten, der links fehlte, so dass beide Schläfelappen sich so zu sagen ergänzten (man vergl. l. c. die Zeichnungen). Leider scheint nicht untersucht worden zu sein, wie Patient sich einzelnen Tönen gegenüber verhielt, auch ist aus der Krankengeschichte nicht klar zu ersehen, ob er auch die innerlichen Klangbilder der Melodien verloren hatte, oder ob diese erhalten waren. Es bleibt daher zweifelhaft, ob die Musiktaub. heit auf Zerstörung des Centrums oder der zuleitenden Bahn beruhte. E dgren schliesst aus seinem Fall, dass die Tontaubheit, wie er das in Rede stehende Krankheitsbild nennt, mit einem hohen Grade von Wahrscheinlichkeit in der ersten oder der ersten und zweiten Windung des linken Schläfelappens vor der Stelle, dessen Verletzung Worttaubheit hervorruft, localisirt sei. Ich kann mich dem nur anschliessen, vorausgesetzt, dass der Begriff Centrum in dem von mir oben angewandten Sinne genommen wird.

8. Beobachtung von Bernhardt (Centralblatt für Nervenheilkunde. Bd. V. S. 249).

46 jähriger Patient, an Mitralstenose leidend. October 1891 plötzliche Bewusstlosigkeit von kurzer Daner, mit rechtsseitiger Hemiparese und ,schwerer" Sprache. S Tage darauf plötzlich grosse Unruhe, versteht seine Umgebung nicht. Status praes. 2 Monate (6. December) später: Kann lesen und versteht, was er liest (liest laut aus einer Zeitung vor). Er hört Töne und Geräusche, aber er versteht nicht, was man zu ihm spricht. „Ich höre jeden Ton, aber das Wort kann ich nicht verstehen, wie es eigentlich heisst", „ich höre alles, aber der Gedanke, der Ton davon, wie man sagt, fehlt immer davon". Er spricht, verwechselt aber dabei oft die Worte oder wenigstens einzelne Buchstaben. Den Gebrauch vorgezeigter Dinge kennt er, die Namen derselben bringt er nur theilweise richtio heraus. Ob die von ihm gemachten Schreibfehler mehr auf seine mangelhafte Bildung zurückzuftihren oder als Paragraphie aufzufassen seien, ist schwer zu unterscheiden. Spricht man Worte vor, so bemüht er sich, richtig nachzusprechen, es wird aber oft falsch: er sieht dabei aufmerksam auf den Mund des Vorsprechenden. Flüsterstimme hört er beiderseits auf viele Fusse; er merkt, dass man spricht, kann aber nichts nachsagen. Es werden ihm die Anfangsverse von „Heil Dir im Siegerkranz" und von der "Wacht am Rhein" vorgesungen. "Er antwortet „Erst geht's hoch, dann tief, dann wieder hoch u. s. w.", d. h. er ist sich wohl der wechselnden Intensität des Klanges, nicht aber der Melodie bewusst. Die ohrenärztliche Untersuchung ergab die Abwesenheit von jedem Ohrenleiden. Nach Verlauf von 20 Wochen war eine wesentliche Aenderung des Krankheitsbildes nicht eingetreten. Ende December hatte die Paraphasie etwas nachgelassen; sieht er einem auf den Mund, und spricht man nicht zu schnell, so versteht er etwas. Drei Wochen später wurde notirt: wenn man eindringlich, aber nicht mit erhobener Stimme spricht (lautes Sprechen, Schreien berührte ihn sehr nnangenehm), so versteht er einiges (ob er dabei auch den Mund fixiren musste, ist nicht angegeben). - Meiner Meinung nach handelte es sich bei dem Kranken um subcorticale sensorische Aphasie in Verbindung mit Leitungsaphasie. 
9. Beobachtung von Sérieux (Revue de médecine. 1893. p. 733, cit. nach $\mathrm{Edg} \operatorname{cin}$, l. c. p. 36 ).

51 jährige Wittwe. Seit 4 Jahren Schwierigkeit, das gesprochene Wort zu verstehen, obwohl sie Geräusche auffassen kann. Jetzt fehlt die Auffassung des gesprochenen Wortes fast völlig. Sie sagt selbst, dass sie die Worte sehr gut höre, aber sie nicht verstehe. Rechts das Gehör infolge einer alten Otitis aufgehoben, links normal, sie reagirt gegen die kleinsten Geräusche. Die gewöhnlichsten Melodien werden nicht erkannt. "Au claire de la lune" wird fur einen Trauermarsch gehalten, die Marseillaise bewirkt eine andächtige Stellung, die lustigsten Melodien werden für Kirchengesänge gehalten. Sie sprieht lebhaft, geringer Grad von Aphasie, schreibt spontan, wie sie spricht; auf Dictat schreibt sie viel schlechter (natürlich, da sie die Worte kaum versteht!). Sie liest und versteht Geschriebenes und Gedrucktes fast völlig correct. Schwierigere Worte kann sie nur nachsagen, wenn sie sie geschrieben gesehen hat. Der Originalaufsatz war mir nicht zugänglich, so dass ich nicht weiss, ob die Beschreibung dort deutlicher ist. Es macht jedoch den Eindruck, als gehöre der Fall hierher.

\section{Ueber einige Einwände gegen das Lichtheim'sche Krankheitsbild.}

Gegen die Lichtheim'sche Lehre von der subcorticalen sensorischen Aphasie sind jüngst von verschiedenen Seiten Einwendungen erhoben, auf die ich bier kurz eingehen will. Zunächst hat Freud ${ }^{1}$ ) die Lehre von der Aphasie einer kritischen Besprechung unterworfen, auf Grund deren er die gesammten Lichtheim-Wernicke'schen Ansichten verwirft und an deren Stelle eine neue Theorie setzt. Er nimmt ein zusammenhängendes Rindengebiet zwischen den Endstätten des N. opticus, acusticus und denen der motorischen Hirnund Extremitätennerven in der linken Hemisphäre an, innerbalb dessen die Associationen und Uebertragungen, auf denen die Sprachfunctionen beruhen, in einer dem Verständniss nicht näher zu bringenden Complicirtheit vor sich gehen. Die psychischen Elemente des Sprachvorganges an bestimmte Stellen dieses Gebietes zu localisiren, lehnt er ab. Die durch die Pathologie scheinbar nachgewiesenen Centren entstehen nur dadurch, dass an diesen Stellen das Associationsgebiet der Sprache an die Rindenfelder jener oben erwähnten Nerven stösst, die hier in dasselbe eingehen. Eine an diesen Stellen sitzende Läsion muss den Zufluss der optischen, akustischen u. s. w. Elemente abschneiden und dadurch den Schein von sogenannten Sprachcentren erwecken. Physiologisch sind sie es aber nicht, da in ihnen keine anderen oder bedeutsameren Vorgänge stattfinden als in den ubrigen Theilen des Sprachfeldes. Demnach giebt es für

1) Zur Auffassung der Aphasien. Leipzig und Wien 1891. 
Freud keine "Centren-" oder corticale Aphasien. Jede Aphasie berubt auf Associations-, also auf Leitungsunterbrechung, sie beruht ferner stets auf Störung in der Hirnrinde selbst, da das Sprachengebiet keine ihm eigenthümlichen zu- und ableitenden Bahnen besitzt, die bis zur Körperperipherie reichen, eine Annahme, die er freilich für den motorischen Theil des Sprachapparates später wieder modificirt.

Mit dieser Freud'schen Lehre steht nun die subcorticale sensorische Aphasie Lichtheim's in unlösbarem Widerspruch. Psychologisch nämlich ist erstere völlig beherrscht von den Klangbildern, an welche einmal die Objectvorstellung, dann das Bewegungsbild und endlich das Lese- mit dem Schreibbild sich angliedern. Nun können die Klangbilder aber nur dann ausfallen, wenn das Rindenfeld des Acusticus rechts und links, und damit der akustische Zufluss zum Associationsfeld der Sprache gestört ist. Dann müssen aber natürlich gleichzeitig andere Hörstörungen ausser der Sprachtaubheit vorhanden sein. Liegt die Zerstörung diesseits der akustischen Rindenfelder, so muss sogar völlige Taubheit entstehen. Findet sich nun ein Fall, in dem das ubrige Gehörsvermögen völlig intact ist, während vollkommene Worttaubbeit bei ungestörtem Vermögen zu sprechen, zu lesen und zu schreiben besteht, so ist er nach der Freud'schen Theorie vollkommen unerklärlich. Dieser Unmöglichkeit ist Freud sich durchaus bewusst. Deshalb sucht er den Lichtheim'schen Fall anders zu deuten. Er vergleicht ihn mit dem Fall von Adler ${ }^{1}$ ), in dem letzterer eine Combination von subcorticaler und transcorticaler sensorischer Aphasie angenommen hatte, und kommt zu dem Ergebniss, dass erstens in beiden Fällen wahrscheinlich eine gemeine peripherisch oder central bedingte Taubheit nicht ohne Einfluss auf das Krankheitsbild geblieben sei, dass zweitens bei beiden Kranken das Bild der subcorticalen sensorischen Aphasie erst nach wiederholten Anfällen von Gehirnerkrankung entstanden sei, welche die Annahme von doppelseitigen Herden zuliessen, dass also wahrscheinlich doppelseitige Läsionen des Hörfeldes neben der peripheren Erkrankung Ursache davon seien.

Nun habe ich aber oben bereits hervorgehoben, dass die in Betracht kommende Lichtheim'sche Bahn nur streckenweise eine einfache ist, sich dann aber in eine von der rechten und eine von der linken Hemisphäre kommende theilt, und dass daher unter Umständen das Krankheitsbild erst durch doppelseitige Herde perfect wird.

1) Siehe oben Fall 3. 
Lich theim hat allerdings diese Möglichkeit nicht besonders erwähnt, aber sie ist eine nothwendige Folge seiner Darstellung. Ich halte also den hierauf beziuglichen Einwand für nicht schlagend. Die Kritik ferner, die Freud an dem auffallenden Verhalten des Lichtheimschen Patienten ausuibt, welches von dem anderer Worttauber ganz abweichend sei, so dass Fre u d darin sogar ein gewisses vorsätzliches Benehmen, den Anschein eines völlig Worttauben erwecken zu wollen, findet, während der Kranke doch etwas hätte hören können, dürfte 'durch die oben gegebene Erklärung von der Nothwendigkeit eines solchen verschiedenen Verhaltens erledigt sein.

Aber auch wenn die Worttaubheit, wie bei meinem Patienten, durch eine geringe Paraphasie complicirt ist, kann das Freud'sche Schema keine Erklärung geben. Man mag die Läsion in dem Sprachfeld hin- und herschieben, wie man will, man findet keine. Zieht man aber functionelle Momente in Betracht und berücksichtigt die von Freud angenommenen Bastian'schen Grade der Erregbarkeit, so ergiebt sich bei deren Anwendung auf den akustischen Bestandtheil des Sprachapparates, der bei unserem Kranken ja betroffen sein muss, Folgendes: gerade die willkürliche Anregung desselben, die am leichtesten hätte versagen müssen, war erhalten oder nur wenig gestört (spontane Sprache, willkürliches Nennen von Gegenständen u. s. w.), die periphere sensible Anregung aber, die der Theorie nach am schwersten versagt, war ganz gestört. Demnach bin ich der Meinung, dass unser Fall, bei dem ferner das Gehörorgan ganz sicher intact war, dem Verständniss wohl zugänglich ist auf Grund der Lichtheimschen Annahme, nicht aber auf Grund der Freud'schen. Nur sind dabei die Anschauungen über die Centra ein wenig zu verändern, und die Leitungsbahnen brauchen nicht gerade in der weissen Substanz, sie können auch in der grauen verlaufen. Wenn die Freudsche Lehre richtig wäre, so müsste bei der Worttaubheit das Krankheitsbild ungefähr immer dem Bilde entsprechen, welches der oben erwähnte Kranke von Pick zeigte (Fall 3).

Denselben Gedanken wie theoretisch Freud, nämlich, dass zu dem Krankheitsbild der Worttaubheit vielleicht eine central bedingte Taubheit beitragen könne, hat gleichzeitig im Anschluss an zwei Beobachtungen $\mathrm{Pick}^{1}$ ) geäussert, ohne dass er jedoch deswegen zu einer Verwerfung der Lichtheim'schen Anschaungen kommt.

Am wichtigsten in dieser Beziehung ist eine jüngst erschienene Schrift von Freud ${ }^{2}$, die sich ausfuhrlich mit den Beziehungen

1) Archiv für Psychiatrie. Bd. XXIII. S. 902.

2) Labyrinthtaubheit und Sprachtaubheit. Wiesbaden 1895. 
zwischen Labyrinthtaubheit und Worttaubheit befasst. Sie bringt zunächst die ïberraschende Mittheilung, dass ein früher von Wernicke ${ }^{1}$ ) beobachteter und kurz als subcorticale sensorische Aphasie beschriebener Fall sich bei einer erneuten Untersuchung als von einer Labyrintherkrankung herrührend erwiesen habe. Ferner wird ein ähnlicher Fall neu beschrieben.

Letzterer Patient (Freud's Fall 1), dessen Anamnese als Ursache eine Meningitis cerebro-spinalis mit Doppelbildern, Schwäche im rechten Arm, Schwindel, Gleichgewichtsstörungen und zeitweiligen Geräuschen im Ohr aufwies, war links völlig taub, rechts war das Sprachverständniss fast völlig aufgeboben, indem er nur bei passender Versuchsanordnung manchmal das Vorgesprochene richtig oder annähernd richtig auffasste, besonders gut Zahlen. „Ich höre blos den Tonfall des Wortes, und dann muss ich nachdenken, was das sein kann", sagte er. Von den sprachlichen Lauten verstand er nur die Vokale a und e sicher, von den Consonanten nur $p$ und $h$ in Verbindung mit a gesprochen, stumm und ohne angehängten Vokal gesprochen sind sie sämmtlich völlig unverständlich. Dagegen hatte er ein gutes Unterscheidungsvermögen selbst für relativ leise Geräusche; musikalische Instrumente unterscheidet er fast stets richtig von einander. Einzelne Töne erkennt er und differenzirt sie bis zu Intervallen von einem ganzen Ton. Für ganz hohe Töne bestand ein Defect. Von bekannten Volksweisen erkennt er nur solche, die einen charakteristischen Rhythmus besitzen. Auch sein musikalisches Gedächtniss hatte gelitten („durch meine Krankheit habe ich viel vergessen, frther kannte ich viele Melodien"), auch war es an verschiedenen Untersuchungstagen verschieden. Mitunter zeigte er sogar im Laufe einer Untersuchung eine temporäre Insufficienz für e in e Melodie, insofern einige Minuten später dieselbe Melodie in seiner Erinnerung wieder auftanchte. Seine Sprache klang raub, hart und laut wie bei einem Schwerhörigen, er sprach fliessend, keine Spur von Paraphasie, Aphasie u. s. w. Die Ohrspiegeluntersuchung ergab keinen Befund, die Stimmgabelprüfung keine verwerthbare Resultate, durch Luftleitung wurden Stimmgabeltöne überhaupt nicht percipirt.

In dem 2. Fall von Freud, der identisch ist mit dem Wernicke'schen Fall von subcorticaler sensorischer Aphasie, wird die Erkrankung zurückgeführt auf einen Sturz auf den Hinterkopf. Patient war starker Potator, hatte öfters Delirium, Krämpfe, ein Mal auch Anzeichen von rechtsseitiger Hemiplegie. Die von Freud

1) Fortschritte der Medicin. 1876. S. 474. 
neuerdings angestellte Untersuchung ergab: absolute Taubheit des rechten Obres, Ausfall der hohen Töne links. Geringe Aufmerksamkeit für Schalleindrücke. Händeklatschen und das Klingen der Glocke hörte er noch auf $1 \mathrm{M}$. Genauere Prüfung unmöglich wegen geringer und schnell abnehmender Aufmerksamkeit. Er sagt: „ich höre alles; ich verstehe nur nicht die Sprache". Stumm gesprochene Consonanten blieben ihm völlig unverständlich. Von den Vokalen versteht er stets a, andere wechselnd. Bei passender Versuchsanordnung versteht er einzelne Worte richtig oder annähernd richtig; einer Unterhaltung mit Verständniss zu folgen, ist ihm dagegen unmöglich. Melodien erkennt er und kann sie nachsingen. Töne benennt er richtig, er unterscheidet sogar noch Vierteltöne, nur die höchsten fehlen ganz. Sprache ganz intact, ebenso Lesen und Schreiben. Eine Mittelohrerkrankung ist nicht vorhanden. Aus der acht Jahre früher aufgenommenen Krankengesehichte ist bemerkenswerth, dass er damals vorgesungene oder vorgepfiffene Melodien nicht auffasste, sie aber singen konnte, wenn man ihm die Melodien aufschrieb. Einzelne direct ins Ohr gerufene Worte verstand er auch damals, ebenso war der Defect für hobe Töne vorhanden. In früheren Krankheitsberichten - er war wegen Delirium häufig im Krankenhause - war übrigens die Erkrankung, wie sich jetzt herausstellt, ganz richtig auf eine solche des Gehörs bezogen worden. Etwas wunderlich ist es daher, wenn man bei Freud liest, die sensorische Aphasie sei damals nicht als solche erkannt worden; denn Freud widerruft jetzt ausdrücklich für diesen Fall die frühere Wernicke'sche Diagnose einer subcorticalen sensorischen Aphasie und fübrt beide Fälle auf doppelseitige Labyrintherkrankung zurïck. Bei beiden war die Krankheit aufgetreten unter Schwindel und Gleichgewichtsstörungen, eine Mittelohrerkrankung liess sich mit Sicherheit ausschliessen.

Obschon wir mit der Diagnose einer Labyrintherkrankung in beiden einander so ähnlichen Fällen einverstanden sind, so können wir doch einige Bedenken nicht unterdrücken, ob nicht in beiden Fällen gleichzeitig complicirende Gehirnstörungen vorhanden waren. Man versteht nämlich nicht, warum der erste Kranke, der Töne ganz gut, abgesehen von den hohen, auffasst, niebt ebenso gut wie der zweite $\mathrm{Me}$ lodien hören kann und zur Erklärung dafür, dass er nur einen relativ kleinen Melodienschatz besitzt, von sich selbst sagt: „Durch meine Krankheit habe ich viel vergessen, früher kannte ich viele Melodien“. Weshalb ist ferner sein musikalisches Gedächtniss, wie es in der Krankengeschichte heisst, an verschiedenen Tagen verschieden? Warum wusste er sogar mitunter Melodien nicht, während sie einige Minuten 
später wieder in seiner Erinnerung auftauchten? Diese Störungen werden zweifellos durch eine Labyrinthaffection nicht, sondern nur durch eine cerebrale Störung erklärt. Es ist also nicht richtig, wenn Fre u $\mathbf{d}^{1}$ ) mit besonderem Nachdruck darauf hinweist, dass bei beiden Patienten von einer Beeinträchtigung des Gedächtnisses nicht gesprochen werden könne. Für den ersten Fall widerlegt ihn die von ihm selbst gegebene Krankengeschichte, mindestens soweit es sich um das musikalische Gedächtniss handelt. Alsdann aber muss man sich in Hinblick anf andere Fälle, wo Sprach- und Melodienverständniss ähnliche parallele, sicher auf das Gehirn zu beziehende Störungen aufwiesen, doch fragen, kommt nicht ein Theil des mangelnden Sprachverständnisses auch hier auf Rechnung einer Gehirnstörung, zumal da eine Meningitis mit Doppelbildern und Schwäche im rechten Arm vorausgegangen war?

Auch der zweite Kranke zeigte ein solches auffallendes Symptom wenigstens in früheren Jahren, wo er vorgesungene oder vorgepfiffene Melodien nicht auffassen, sie nicht oder nur mit grosser Entstellung nachpfeifen konnte, während er Töne - von den hohen abgesehen gut unterschied. Wenn nach Freud der Kranke Worte nicht verstand, weil er infolge des Nichtrerstehens der Consonanten entstellte Klangbilder von ihnen erhielt, so hätte er umgekehrt Melodien doch verstehen müssen, da er deren Bestandtheile, die einzelnen Töne, richtig erkannte. Es liegen also auch für den zweiten Fall Bedenken gegen den Ausschluss einer für das Sprachverständniss in Betracht kommenden Gehirnerkrankung wenigstens aus einem früheren Stadium vor.

Kann nun eine Labyrintherkrankung allein den beschriebenen Einfluss auf das Sprachverständniss haben? Sichere Beweise konnte Freud hierfür nicht finden, gewann aber zunächst durch Untersuchungen an Ohrenkranken die Ueberzeugung, dass das Betroffensein beider Labyrinthe fur das Zustandekommen der vorliegenden Form von Sprachtaubheit ein nothwendiges Postulat sei. Zur weiteren Entscheidung der Frage untersuchte er dann eine Anzahl von mit Sprachresten begabten Taubstummen, deren Erkrankung mit Wahrscheinlichkeit auf Labyrinthleiden zu beziehen war und fand, dass sie ganz äbnliche Erscheinungen zeigten. Für die gewöhnliche Unterhaltung bestand vollständige Sprachtaubheit, bei passender Versuchsanordnung wurden öfters einzelne Worte verstanden. Der Grund war auch hier, dass zuweilen zwar Vokale, nicht aber Consonanten deutlich unterschieden werden konnten, und dass daher von den Wörtern unreine Klangbilder aufgenommen wurden.

1) l. c. S. 38 . 
Freud kommt daher zu dem Schlusse, dass der klinische Begriff der "Sprachtaubheit" (resp. Worttaubheit resp. sensorische Aphasie) unzulänglich und zwar zu eng gefasst sei. Das entsprechende Symptomenbild sei nicht ausschliesslich der Ausdruck für eine Läsion, welche die Function des N. acusticus innerhalb seiner centralen Endausbreitung in der Rinde des Schläfelappens oder auf seinen „subcorticalen" Wege durch das Marklager des Grosshirns beeinträchtige, auch unter dem Einfluss von Gehörleiden, welche extracerebral peripherwärts localisirt seien, könne Sprachtaubheit zur Entwicklung gelangen.

Freud glaubt sogar den verschiedenen Formen der Sprachtaubheit eine einheitliche physiologische Erklärung unter Bezug auf Experimente von Bleuler zu Grunde legen zu können und kommt so zu dem Schlusse, bei der Sprachtaubheit handle es sich um ein Symptom von Seiten des acustischen Apparates, welches keinen absoluten topischen Werth besitze; die veranlassende Läsion könne im Gehirn, im Acusticusstamm oder im Labyrinth, ja sogar unter Umständen im Mittelohr localisirt sein und den gleichen Functionsausfall veranlassen. Es will mir scheinen, als ob hier etwas bewiesen wird, was nie bestritten worden ist; denn Sprachtaubheit, Worttaubheit und sensorische Apbasie sind doch meines Erachtens keineswegs in dem Sinne Synonyma, in welchem Freud sie hier anwendet. Besonders ist Sprachtaubheit wohl niemals als ein nur bei sensorischer Aphasie auftretendes Symptom betrachtet worden. Ich selbst behandelte um dieselbe Zeit, wo ich den oben beschriebenen Fall in Beobachtung hatte, einen Patienten mit Ohrensausen, der ganz eclatante Sprachtaubheit aufwies bei erhaltenem Gehör für Geräusche. Ich bin nie auf die Idee gekommen, diesen Fall wegen der Worttaubheit für eine sensorische Aphasie zu halten; schon die ganze Entstehung wies mit Sicherheit auf ein Ohrleiden hin, und in allen Lehrbüchern der Ohrenheilkunde findet man die Angabe, dass bei Ohrenkranken mitunter zwischen dem Gehör für die Sprache und dem für Geräusche die grössten Unterschiede vorkommen können.

Uebrigens beweisen die Experimente Bleuler's, wie ich meine, keineswegs das, was sie beweisen sollen. Bekanntlich schliesst er aus der Beobachtung, dass man zwar noch die in einem Nebenzimmer entstehenden Geräusche deutlich erkennen, nicht aber die dort gesprochenen Worte verstehen könne, was er eine experimentelle incomplete Worttaubheit oder Wortschwerhörigkeit nennt, dass 1. die Worttaubheit nicht nothwendig auf eine centrale Läsion zurtickzufibren ist, und dass 2. eine allgemeine Gehörstörung das (akustische) Ver- 
stehen der Worte aufheben kann, ohne dass die Wabrnehmung anderer Schallqualitäten erheblich alterirt ist. Nun haben aber die Sprachlaute bekanntlich eine ausserordentlich verschiedene Hördistanz infolge ihrer verschiedenen Klangfarbe und Tonstärke. Nach den Untersuchungen von Wolff bilden die Grenzen a mit einer Hördistanz von $360, p$ und $b$ mit einer solchen von 18 und $h$ (als verstärkter Hauch) mit einer solchen von nur 12 Schritten. Demnach kommen von einem in der Ferne gesprochenen Wort gar nicht alle Buchstaben in das $\mathrm{Ohr}$, ebenso natirlich auch nicht aus einem abgeschlossenen Nebenraum, da die Wände die Buchstabengeräusche abschwächen. Fread hörte in der That bei einer Wiederholung der Bleulerschen Experimente, je weiter der Sprechende sich von der Zwischenthur des Nebenzimmers entfernte und je leiser er sprach, schliesslich nur noch unklare Vokale, die an a und o anklangen. Dies sind aber nach Wolff's Untersuchung gerade die beiden tonkräftigsten Sprachlaute. Es gelangt somit bei dem Bleuler'schen Experiment ein Etwas in das Ohr, das schon physikalisch dem gesprochenen Worte nicht mebr entspricht, weil ein Theil der Schwingungen unterwegs ausfällt. Wie soll nun da das Ohr es anfangen, trotzdem das gesprochene Wort za verstehen? Es könnte ebenso gut Jemand einem Anderen ein beliebiges Geräusch unmittelbar ins Ohr flüstern und nun von ihm verlangen, dass er ein Wort versteht. Es handelt sich dabei weder um eine experimentelle Wortaubheit noch um Wortschwerhörigkeit, sondern es liegt nur ein im Princip verfehltes Experiment vor, dessen Zusammenhang mit dem vorliegenden Thema mir nicht einleuchten will.

Hat: nun Freud, indem er in zwei Fällen den Nachweis erbrachte - wir seben dabei von den obigen Bedenken ab - dass Sprachtaubheit durch doppelseitige Labyrintherkrankung entstehen könne, die Existenz des Li ch theim'schen Krankheitsbildes der subcorticalen sensorischen Aphasie erschittert?' Oder sind beide nicht vielmehr ganz verschiedene, klinisch gut zu unterscheidende Erkrankungen, unterscheidbar schon durch Verschiedenheiten der in beiden Fällen das Hauptsymptom bildenden Sprachtaubheit? Wenn oben subcorticale sensorische Aphasie und Sprachtaubheit als nicht identische theoretische Begriffe hingestellt wurden, so setzt das freilich zunächst eine Verständigung über die Nomenclatur voraus etwa in demselben Sinne, wie eine solche über den Begriff der motorischen Aphasie nöthig ist. Lichtheim reservirt den letzteren, wie mir scheint, mit Recht für diejenigen Formen der Sprachlosigkeit, welche nicht von Störungen der Articulationsmechanik begleitet sind, während 
Wernicke auch die Sprachlosigkeit, welche im Endstadium der progressiven Bulbärparalyse durch vollständige Zerstörung der Bulbärkerne entsteht, zur Aphasie rechnet. Zur subcorticalen sensorischen Aphasie wird man gleicher Weise, den Intentionen des ersten Beschreibers derselben, wie ich glaube, folgend, nur Störungen der zu dem sensorischen Sprachcentrum unmittelbar zuleitenden Bahn ( $W T_{1}$ und $W T_{2}$ ) rechnen können, nicht aber diejenigen Störungen, die beim Hören der Sprache auftreten, welche auf Läsion der allgemeinen Hörsphäre oder der darunter liegenden Apparate beruben.

Giebt es nun bei verschiedenem Sitz der Läsion verschiedene Formen von Sprachtaubheit, die sich deutlich von einander trennen lassen, etwa so, wie man das in der obigen Parallele durch die Begriffe der Aphasie und der Anarthrie kann? Ich glaube das auf Grund der jetzt vorliegenden Beobachtungen bejahen zu miissen, und zwar ist das entscheidende Kriterium genau dasselbe wie nach Lichtheim bei der Unterscheidung zwischen Aphasie und Anarthrie, nämlich das eine Mal tritt der verbale Charakter der Störung, das andere Mal die Läsion der Laute, der Sprachelemente in den Vordergrund.

Bei der subcorticalen sensorischen Aphasie werden niemals irgend welche Wörter verstanden, man mag den Versuch (langsames, deutliches Sprechen u. s. w.) einrichten, wie man will; denn die Association der Buchstabenlaute zum Wortklang ist völlig aufgehoben. Bei der anf Labyrintherkrankung beruhenden Sprachtanbheit, die wir als acustische bezeichnen wollen, werden dagegen immer noch mehr oder weniger zahlreiche Wörter, besonders bei passender Versuchsanordnung, verstanden; denn das Gefühl für den Rhythmus und die Betonung der Wörter, die Anzahl ihrer Silben ist vorhanden, da die Laute, soweit sie gehört, auch associirt werden, nur ist das Associationsproduct nicht das richtige, da eine Anzahl der zugehörigen Elemente nicht zur Empfindung gelangten. Die Kranken suchen dann bei sich nach ähnlich lautenden Wortklangbildern, die ihnen innerlich ja nicht fehlen, und bemühen sich das Vorgesprochene za errathen, wobei sie natürlich bäufig falsch rathen. Bei der sensorischen Worttaubheit werden sämmtliche Sprachlaute, Vokale und Consonanten, einzeln richtig und ohne Schwierigkeit gehört, besonders ist dies auch mit den stumm gesprochenen Consonanten der Fall; denn das Centrum für die Association der Bachstaben aus den versebiedenen sie zusammensetzenden Gehörsempfindungen ist ja intact und zugänglich. Bei der acustischen Sprachtaubheit werden stumm gesprochene Consonanten überhaupt nicht, mit Vokalen gesprochene 
nur zuweilen und auch Vokale nicht immer richtig verstanden; je besser im einzelnen Fall das Verständniss der Sprachlaute ist, desto besser ist auch das Wortverständnis. Ist die sensorische Aphasie nicht vollständig (Fall Schmidt), weil die Associationsbahn nur beschädigt, nicht vernichtet ist, so kann ein einsilbiges Wort verstanden werden, indem man bei der Aussprache die einzelnen Buchstaben scharf von einander trennt, sodass sie deutlich hervortreten. Bei mehrsilbigen Wörtern muss man das für jede Silbe wiederholen. Die successive Association gelingt noch, weil nun bei der langsamen Aussprache die einzelnen Buchstaben ordnungsgemäss die gestörte Leitungsbahn zum Wortassociationscentrum passiren können, während sie bei der gewöhnlichen fliessenden Aussprache in Wirrwarr durch einander kommen. Demgegenüber fassen die acustisch Worttauben die Wörter am besten auf bei möglichst reiner, scharf accentuirter Aussprache.

Bei der subcorticalen sensorischen Apbasie ist die Sprache in ihrem Timbre nicht verändert; bei der acustischen Worttaubheit bekommt sie ein rauhes, hartes und lautes Timbre wie bei einem Schwerhörigen. Bei Freud's erstem Fall zeigte sich das schon $4^{1 / 2}$ Monat nach Beginn der Erkrankung. Auch mein eigener oben erwähnter Patient mit acustischer Worttaubheit sprach auffallend laut und hart und unterschied sich dadurch aufs deutlichste von jenem anderen sensorisch Aphasischen.

Mebr als ein Zufall dürfte es wohl sein, dass in sämmtlichen oben der subcorticalen sensorischen Aphasie zugerechneten Fällen das Verständniss für die Musik fehlte - nur im Falle von Schmidt findet sich darüber keine Angabe - während einzelne Töne gehört wurden. Also auch hier fehlt das Vermögen der successiven Association. Die vorgesungenen, bekannten oder unbekannten, Melodien konnten auch nicht nachgesungen werden. Ganz im Gegensatz dazu konnte der Freud'sche Fall II nicht nur ihm bekannte Melodien erkennen und nachsingen, er sang auch ihm unbekannte Melodien richtig nach. Einzelne Töne erkannte er ebenfalls ganz genau, unterschied sogar noch Intervalle von Vierteltönen, hatte aber einen Defect für hohe Töne von $\mathbf{c}^{3}$ an. Ob Lücken in der Tonreihe bei der anderen Gruppe von Kranken vorhanden waren, darüber ist in den Krankengeschichten nichts angegeben; der Theorie nach dirfte es nicht der Fall sein. Unser eigener Kranker entsprach dem. Einen Defect für hohe Töne batte auch der Patient I von Freud, er erkannte aber nur vereinzelte Melodien, die meisten nicht. Einiges, wie die Wacht am Rhein, erkannte er nicht, trotzdem es ihm bekannt 
vorkam, pfiff es aber nach. Ich habe bereits oben begrïndet, weshalb ich eine centrale Ursache bei ihm als mitwirkend ansehe, sodass der Fall den durchgehenden Unterschied, der mir zwischen beiden Gruppen im Verhalten gegen Musik vorhanden zu sein scheint, nicht beseitigen kann. Dennoch bin ich der Meinung, dass die Musiktaubheit nur eine Begleiterscheinung der sensorischen Sprachtaubheit ist, aber nicht nothwendig dazu gehört; denn es sind ja schon Fälle beschrieben, wo sie ohne letztere auftrat. ${ }^{1}$ ) Die fur diese Associationen erforderlichen nervösen Apparate dürften also nicht identisch sein, sondern nur eine einander ganz benachbarte Lage haben. Zu diesem Schluss kommt ja auch Edgren ${ }^{2}$ ) in seinem oben erwähnten Aufsatz.

Auch beim Versuch nachzusprechen dürften zwischen beiden Krankheitsgruppen Unterschiede hervortreten. Mein Kranker war dazu, obschon er sich die grösste Mühe gab, durchaus ausser Stande. Ebenso scheint es im Lich theim'schen Fall sich verhalten zu haben. Man begreift auch leicht diese Unmöglichkeit, da die Kranken ein vollkommen wirres Geräusch hören. Freud's Patient I konnte dagegen, obwohl er es sehr ungern that, die Worte so nachsprechen, wie er sie gehört hatte, und das Nachgesprochene hatte, wenn es auch sinnlose Worte waren, doch immer einen sprachlichen Charakter.

Ferner unterschieden sich beide Gruppen durch die begleitenden Momente. Zunächst muss natürlich bei der sensorischen Aphasie das Gehör functionell, abgesehen vom Sprachverständniss, ganz intact sein, und war es auch in den genau untersuchten Fällen (Kahler and Pick, eigener Fall), was besonders gegenüber den Freud'schen Ausftihrungen scharf hervorgehoben werden muss. Dessen Angabe, Lichthei m erwähne die Möglichkeit, dass sein Kranker , in leichtem Grade taub" genannt werden könne, habe ich in dem Lichtheim schen Aufsatz nicht auffinden können. Dagegen werden sich bei der Labyrinthworttaubheit wohl immer irgend welche Defecte ausser dem mangelnden Sprachverständniss nachweisen lassen. In den Freudschen Fällen bestand z. B. Verlust des Hörvermögens für hohe Töne, aufgehobene Knochenleitung, ja beide waren sogar auf einem $\mathrm{Ohr}$ ganz taub. Andererseits finden sich bei der subcorticalen sensorischen Aphasie häufig weitere aphasische Störungen wie Paraphasie, Paragraphie u. s. w., Störungen, die sofort den Verdacht eines cerebralen Ursprungs der Worttaubheit erregen müssen, während sie natïrlich bei Labyrinthaffectionen fehlen.

1) Vgl. auch den Fall von Brazier, cit. bei Edgren, Deutsche Zeitschrift für Nervenheilkunde. Bd. VI. S. 40.

2) S. oben Fall 7. 
Dennoch soll nicht geleugnet werden, dass in manchen Fällen eine sichere Erkennung dessen, was vorliegt, nicht möglich sein wird, z. B. wenn zu einem mit Sprachtaubheit verbundenen Ohrleiden eine subcorticale sensorische Aphasie binzutritt u. s. w. Aehnliche Schwierigkeiten finden sich aber auch anderswo, z. B. bei der Diagnose der subcorticalen motorischen Aphasie. So erwähnt Lichtheim die Schwierigkeit der Erkennung einer solchen, wenn dazu eine Pseudo-Bulbärparalyse hinzutritt; dann wird nur eine ganz genaue Anamnese resp. die genaue Kenntniss der Entstehung der Affection mitunter eine klare Erkenntniss dessen, was vorliegt, ermöglichen.

Den Verlauf, nämlich dass keine Ruickbildung stattfindet, baben beide Affectionen mit einander gemein. Findet eine solche bei der subcorticalen sensorischen Aphasie statt, so kann es sich nur wie in dem Falle von Schmidt um eine indirecte Läsion der Bahn gehandelt haben. Der Verlauf unterscheidet sie ferner zur Genüge von der corticalen und transcorticalen sensorischen Aphasie, die sich, wie alle Autoren, die solche Fälle beobachteten, angeben, meist rasch ausgleicht. Auch zeigen die letzteren Kranken tiberhaupt ein ganz anderes Verhalten, indem sie alle an sie gerichteten Fragen in der Meinung, sie verstanden zu haben, beantworten und zwar in der verkehrtesten Weise, während bei der subcorticalen sensorischen Aphasie die Kranken sich um eine Anrede überhaupt gar nicht kümmern. Ferner sind bei der corticalen sensorischen Aphasie regelmässig die Erinnerungsbilder der Wortklänge in Unordnung gerathen, woraus sich weitere Störungen ergeben, auf die hier nicht eingegangen werden soll. Bei der subcorticalen sensorischen Aphasie sind diese Erinnerungsbilder natürlich intact, und ihr Erhaltensein muss sich durch den erhaltenen Einfluss auf die Sprache u. s. w. nachweisen lassen. Doppelseitige Läsionen beider Hörfelder werden sich von der subcorticalen sensorischen Aphasie wohl stets leicht unterscheiden lassen, da sie kaum eine isolirte Störung des Sprachverständnisses ohne andere Symptome seitens des Gehirns werden machen können. Dadurch wird sich auch die durch sie erzeugte Störung des Sprachverständnisses von der durch Labyrintherkrankung bedingten abgrenzen lassen, wie schon Freud richtig bemerkt.

Dagegen wird sich das Bild in manchen Fällen kaum entwirren oder erkennen lassen, wenn von den in Betracht kommenden Centren und Bahnen mehrere zugleich an der Läsion betheiligt sind. Ob das nicht sogar die Mehrzahl aller Fälle sein wird, muss der weiteren Beobachtung überlassen bleiben. Die reinen uncomplicirten Fälle da- 
gegen lassen meines Erachtens eine sichere Erkenntniss ohne grosse Schwierigkeit zu.

Gegenüber mannigfachen in letzter Zeit geäusserten Bedenken glaube ich, im Vorstehenden nachgewiesen zu baben, dass das von Lich theim angenommene Krankheitsbild der subcorticalen sensorischen Aphasie in Wirklichkeit existirt und mit einigen Abänderungen die aber das Princip der Sache nicht treffen, auch so gedeutet werden muss, wie Lichtheim es gethan hat. Die von Wernicke eingeführte, sehr zweckmässige Nomenclatur zu verlassen, liegt meines Erachtens kein Anlass vor. Sie bedarf nur der Ergänzung. Die durch Obrleiden ( $A_{1}$ und $A_{2}$ ) bedingte Worttaubheit wurde im vorliegenden Aufsatz als akustische, die durch Läsion der Hörsphären $\left(H_{1}\right.$ und $\left.H_{2}\right)$ bedingte als sensorisch-akustische bezeichnet. Sollte das Symptom einmal durch Läsion der Bahnen zwischen $A$ und $H$ entstehen, so könnte man von subcorticaler sensorisch-akustischer Worttaubheit sprechen. Vielleicht findet Jemand eine noch bessere Nomenclatur. 